\title{
IDENTITIES IN THE ALGEBRA OF PARTIAL MAPS
}

\author{
MARCEL JACKSON AND TIM STOKES
}

\begin{abstract}
We consider the identities of a variety of semigroup-related algebras modeling the algebra of partial maps. We show that the identities are intimately related to a weak semigroup deductive system and we show that the equational theory is decidable. We do this by giving a term rewriting system for the variety. We then show that this variety has many subvarieties whose equational theory interprets the full uniform word problem for semigroups and consequently are undecidable. As a corollary it is shown that the equational theory of Clifford semigroups whose natural order is a semilattice is undecidable.
\end{abstract}

\section{INTRODUCTION.}

The variety of agreeable semigroups was introduced by the authors in [12] as an abstraction of some properties of the semigroup of partial maps on a set. As well as the usual multiplication modelling composition there is a second binary operation $*$ giving information on where two elements 'agree'. For a set $X$ we let $\mathcal{P}_{X}$ denote the semigroup of all partial maps on $X$ (acting on the left) under composition. Define $*$ on $\mathcal{P}_{X}$ by letting $f * g$ denote the restriction of the identity map to the elements of $X$ where $f$ and $g$ are defined and agree. In [12], the (isomorphic copies of) subalgebras of this construction are abstractly described and turn out to be a finitely based variety, the so-called twisted agreeable semigroups. While twisted agreeable semigroups are not the first algebraic models of partial maps, they appear to be maximal amongst existing approaches in the sense that the operation $*$ is not a term function in the operations of any previously considered model of partial maps. (On the other hand, many previous models interpret within twisted agreeable semigroups).

The authors arrived at the class of twisted agreeable semigroups via an enrichment of the twisted RC-semigroups considered in [11]. Twisted RC-semigroups also coincide with the type SL2 $\gamma$-semigroups of Batbedat [1], and are the variety generated by the right type-A semigroups studied by Fountain [5]. In [1] and [5], the free algebras in this class are constructed and the equational problem for the variety is solved. The first main result in the current paper is a positive solution to the corresponding problem for the variety of twisted agreeable semigroups. The algorithm substantially extends that of [1] and [5] and turns out to be related to a weakened uniform word problem for semigroups; specifically to testing membership in finitely generated left congruences on free semigroups. As a corollary, we obtain an algorithm describing the equational theory of semigroups of partial maps with the operations of composition and relational intersection-these were shown to form a variety by Garvac'kiū [6]. (These results are established over Sections $3-5$.)

We then contrast this result by showing that a substantial number of related classes of agreeable semigroups interpret the usual uniform word problem for semigroups (equivalently, membership in finitely generated congruences on free semigroups) and so have undecidable equational theory. For example, this holds for the variety of agreeable semigroups whose closed elements are central. We are able to extend the undecidability result to include the variety of naturally semilattice ordered Clifford semigroups (with the unary operation of inverse) as studied by Leech [16], and the variety generated by flat extensions of finite groups. See Sections 6 and 7 .

This paper has a sequel [10] in which the undecidability results of the final sections are shown to be part of a much more general translation of universal Horn classes into varieties.

2000 Mathematics Subject Classification. 08A02, 06F05, 20M20.

Key words and phrases. Agreeable semigroups, algebra of partial maps, word problem, equational problem.

The first author was partially supported by ARC Discovery Project Grant DP0342459. 


\section{Preliminaries}

2.1. RC-semigroups, SLORC's and agreeables. A right closure semigroup (RC-semigroup) is a semigroup $\mathbf{S}$ with unary operation, $C$, satisfying the laws

(1) $x C(x) \approx x$

(2) $C(x) C(y) \approx C(y) C(x)$,

(3) $C(C(x)) \approx C(x)$ and

(4) $C(x y) \approx C(x y) C(y)$.

The 'closed' elements $\{C(x): x \in S\}$ form a subsemilattice $C(S)$ (the law $C(x) C(y) \approx C(C(x) C(y)$ ) follows) with the properties that for every $x$, the element $C(x)$ is the smallest $e \in C(S)$ with $x e=x$; in fact this can be given as a defining property of RC-semigroups. (We note that the left sided version of these structures was previously introduced in [1]; see also [2].) RC-semigroups are a reasonable generalisation of inverse semigroups; on any inverse semigroup the term operation $C(x):=x^{-1} x$ gives rise to an RC-semigroup (the natural $R C$-semigroup on an inverse semigroup).

In the study of RC-semigroups, there are a number of natural subvarieties:

(5) $C(x) y \approx y C(x y)$ (twisted RC-semigroups)

(6) $C(C(x) y) \approx C(x y)$ (the right congruence condition)

(7) $C(x C(y)) \approx C(x) C(y)$ (normal RC-semigroups).

We note that $5 \Rightarrow 6 \Rightarrow 7$ (see $[11,12]$ ). The natural RC-semigroup on an inverse semigroup satisfies the twisted law (and hence the other laws as well). The right congruence condition and normality seem to be required to extend the theory of inverse semigroup congruences, while the twisted law is exactly what is required to extend the Vagner-Preston representation of inverse semigroups:

Proposition 2.1. (See [11]) If $\mathbf{S}$ is a twisted $R C$-semigroup, then $\mathbf{S}$ is isomorphic to a family of partial maps over the set $S$ where multiplication is composition and the closure of a map $x$ returns the restriction of the identity map to the domain $\operatorname{dom}(x)$. Conversely, every semigroup of partial maps with closure defined in this way is a twisted RC semigroup.

The representation in this proposition associates with each $s \in S$ the partial map on $S$ with domain $C(s) S$ and with action $x \mapsto s x$.

We direct the reader to [11] for further details on RC-semigroups and their relatives, but of particular interest are weakly right ample semigroups (see [7] for the left-sided version), and right type-A semigroups (see [5] for example). These closely related classes have seen reasonably extensive research. Weakly right ample semigroups coincide with twisted RC-semigroups with the additional restriction that every idempotent is closed. Right type-A semigroups correspond to a further subclass of the variety of twisted RC-semigroups where again all idempotents are closed. Both of these classes form quasivarieties generating the variety of twisted RC-semigroups (see [2]). Also closely related is the variety of 1-stacks, which are algebras in the operations of multiplication and $\triangleright([18]$; see also Section IV, Example 17 of [19] for defining identities). It is not hard to verify that 1-stacks with identity element are term equivalent to twisted RC-semigroups where $x \triangleright 1 \mapsto C(x)$ and $y C(x) \mapsto x \triangleright y$. A version of Proposition 2.1 is obtained for 1-stack semigroups in [18]. Lastly, we mention the work of Trokhimenko [22] who established an $n$-place function version of Proposition 2.1 (with $n=1$ corresponding to the twisted RC-semigroups).

There is a natural order on any RC-semigroup given by $x \leq y$ if $x=y C(x)$, or equivalently if $x=y C(z)$ for some $z$. This is stable under multiplication on the left and, in the case of twisted $\mathrm{RC}$-semigroups, also on the right. In the case of the natural RC-semigroup on an inverse semigroup, the order agrees with the usual notion of the natural order on an inverse semigroup (given by $x \leq y$ if $x=y e$ for some idempotent $e$ ). The property that this partial order is a semilattice order can be captured equationally if the semilattice operation $\wedge$ is included in the signature. The corresponding structures are called semilattice ordered $R C$-semigroups (or $S L O R C$ 's) and can be defined by the usual RC-semigroup rules plus the axioms:

(8) $a \wedge a \approx a$ and $a \wedge b \approx b \wedge a$;

(9) $a \wedge b \approx a C(a \wedge b)$; and 
(10) $(a \wedge b) \alpha \approx(a \alpha) \wedge(b \alpha)$ for all $\alpha \in C(S)$.

The RC-semigroup condition of normality is equivalent to either of the laws

$\left(7^{\prime}\right)(x \wedge y) \alpha \approx(x \alpha \wedge y)$ or

$\left(7^{\prime \prime}\right)(x \wedge y) \alpha \approx(x \alpha \wedge y \alpha)$.

Proposition 2.1 does not extend to SLORC's that are twisted as RC semigroups unless the right distributive law

(11) $(x \wedge y) z \approx x z \wedge y z$

holds. These are called twisted SLORC's and the representation used for Proposition 2.1 extends to the following.

Proposition 2.2. [12] If $\mathbf{S}$ is a twisted $S L O R C$, then $\mathbf{S}$ is isomorphic to a family of partial maps over the set $S$ where multiplication and closure are as before, while $x \wedge y$ is the intersection of the maps $x$ and $y$ as binary relations. Conversely, every algebra of partial maps with intersection and closure as described is a twisted SLORC.

This proposition also follows from the $n=1$ case of a more general result for multiplace functions obtained by Dudek and Trokhimenko [4].

The variety of agreeable semigroups is a convenient variety which is term equivalent to the variety of SLORC's. On any SLORC, we may define a binary $*$ by $x * y:=C(x \wedge y)$. Under this definition we may directly translate the SLORC axioms into a finite system of identities in $\cdot$ and $*$; these define agreeable semigroups. Conversely, on any agreeable semigroup we may set $C(x):=x * x$ and $x \wedge y:=x(x * y)$ and obtain a SLORC. These translations give a term equivalence of the two varieties. The class of twisted SLORC's translates to the so-called twisted agreeables (denoted by T), which can be defined within semigroups with binary $*$ by the laws:

(12) $a(a * a) \approx a$

(13) $a * b \approx b * a$;

(14) $a(a * b) \approx b(a * b)$

(15) $(a * b) *(c * d) \approx(a * b)(c * d)$

(16) $(a * b)(c * d) \approx a(c * d) * b ;$ and

(17) $(a * b) c \approx c(a c * b c)$.

(To obtain agreeable semigroups whose underlying closure is normal, we only require laws $12-16$.) If the semigroup is a monoid, the first law above can be replaced by $1 * 1 \approx 1$ and the fourth follows from the others and can be omitted. Some useful consequences of laws 12-16 are

$(18)(a * b)(c * d) \approx(c * d)(a * b)$ and $(a * b)(a * b) \approx(a * b)$.

Note that under the appropriate interpretation of $C$ and $\wedge$, all of the laws 1-18 hold in the variety of twisted agreeable semigroups (and follow from laws 12-17).

Proposition 2.3. [12] If $\mathbf{S}$ is a twisted agreeable, then $\mathbf{S}$ is isomorphic to a family of partial maps over the set $S$ where multiplication is composition and for maps $x, y$, the map $x * y$ is the restriction of the identity to the points where $x$ and $y$ are defined and agree. Conversely, every algebra of partial maps constructed in this way is a twisted agreeable.

It follows that every twisted agreeable semigroup is embeddable in a twisted agreeable monoid. We shall solve the equational problem for twisted agreeable monoids, the more general case then following from this observation.

While we concentrate on the agreeable notation, it is occasionally convenient to revert to SLORC notation. In particular, we will often refer to closed elements, which are precisely those of the form $x * y$ for some $x, y$. Indeed, recalling that the closure of $x$ is defined to be $C(x):=x * x$, we get $C(x * y)=(x * y) *(x * y) \stackrel{15}{\approx}(x * y)(x * y) \stackrel{18}{\approx} x * y$. (Here, and elsewhere, numbers written in superscript above $\approx$ symbols indicate which of the laws of this section are used in the equational deduction.) While the axioms 12-17 above may appear somewhat more complicated than the corresponding SLORC axioms, there is a sense in which the $*$ operation is more convenient. It will become clear that the $*$ operation acts in a similar way to the relation of equality - here we have 
in mind that an expression $x * y$ can be (loosely) thought of as representing the statement $x=y$. This intuition is useful in proofs to follow.

There are many other semigroup-related classes for which representations in terms of partial maps have been established. We direct the reader to the excellent survey by Schein [18] for many interesting examples. We here mention two more that are of particular relevance to our approach.

Semigroups of partial maps closed under the operation of intersection were described abstractly by Garvac'kiı [6] who showed that up to isomorphism, they form the variety of semigroups with an additional semilattice operation $\wedge$ satisfying the identities

(19) $(x \wedge y) z \approx x z \wedge y z$ and

(20) $x(a \wedge b \wedge c) \wedge y(b \wedge c) \approx x(a \wedge c) \wedge y(b \wedge c)$.

Because members of this variety (which we call Garvac'kiน semirings) are representable as subalgebras of $\mathcal{P}_{X}$ (for some set $X$ and with the operations of composition and intersection), Proposition 2.2 shows that they are a class of subreducts of twisted SLORC's; that is, subalgebras of reducts of twisted SLORC's to the operations of · and $\wedge$. In Corollary 5.11 we will provide an algorithm for recognising identities in the equational theory of Garvac'kil semirings.

Also of direct interest here are the so-called inverse algebras or naturally semilattice ordered inverse semigroups of Leech [16, 17] (see also [6]). Propositions 2.2 and 2.3 continue to generalise the Vagner-Preston representation for inverse semigroups, so that if $\mathbf{S}$ is an inverse semigroup with the additional operations of $*$ or $\wedge$ inherited from the natural closure, then the corresponding representation gives $\mathbf{S}$ as a system of injective partial maps (the corresponding representation in this case was obtained earlier by Leech in [16]). We also examine these briefly in Section 7 and show that for the Clifford semigroup case (where idempotents are central) the equational theory is undecidable.

Twisted agreeable semigroups interpret many of the existing approaches to modelling the algebra of partial maps. However, the definition of the twisted agreeable $*$ on $\mathcal{P}_{X}$ is based on domain information. Some other approaches to the algebra of partial maps make use of both domain and range information; in particular Schweizer and Sklar (see [20] for example) began a description based on two-sided closure semigroups (under the terminology given above). Twisted agreeables do not interpret these structures but conversely no existing approach interprets twisted agreeable semigroups.

2.2. Uniform word problems, quasi-identities and finitely generated congruences. Recall that a presentation of an algebra in a variety $\mathrm{V}$ is a pair $\langle A: R\rangle$, where $A$ is a set of generators and $R$ is a set of pairs of elements of the $\mathrm{V}$-free algebra freely generated by $A$ (members of $R$ are usually called relations and written as equalities). A presentation uniquely defines the algebra in $\mathrm{V}$ consisting of the $\mathrm{V}$-free algebra freely generated by $A$ and then factored by the congruence generated by the pairs in $R$. An instance of the uniform word problem for $\mathrm{V}$ is a presentation $\langle A: R\rangle$ and a further relation $u=v$. The uniform word problem asks if $u$ and $v$ are equal in $\langle A: R\rangle$. Equivalently, it asks if $u$ and $v$ are congruent under the congruence generated by $R$ on the $\mathrm{V}$-free algebra. We note that for semigroups, we have $u=v$ in $\left\langle A:\left\{u_{1}=v_{1}, \ldots, u_{n}=v_{n}\right\}\right\rangle$ if and only if $u$ can be transformed into $v$ by way of successive replacement of subwords of the form $u_{i}$ by those of the form $v_{i}$ (or $v_{i}$ by $u_{i}$ ) for $i \leq n$.

The uniform word problem in a variety is also equivalent to the quasi-equational problem, which asks when a quasi-identity $\left(\&_{1 \leq i \leq n} u_{i} \approx v_{i}\right) \rightarrow u \approx v$ holds in $\mathrm{V}$ (see [14] for example). The uniform word problem and the quasi-equational problem are also equivalent for pseudovarieties (classes closed under subalgebras, homomorphic images and finite products); here a presentation $\left\langle A:\left\{u_{1}=v_{1}, \ldots, u_{n}=v_{n}\right\}\right\rangle$ and pair $u, v$ constitute a "yes" instance of the uniform word problem if whenever $\mathbf{S}$ is an $A$-generated member of the pseudovariety, and has $u_{i}=v_{i}$ for each $i \leq n$, then also $u=v$.

We direct the reader to [14] for more information on these problems and their relationships. 


\section{THE CONGRUENCE $\cong$}

Let $S$ be a fixed alphabet throughout, with $S^{*}$ the free monoid on the generators $S$.

In this section we give a construction for the free twisted agreeable monoid generated by $S$. Of course, this algebra can be obtained by taking a quotient of the completely free algebra in the operations - and $*$, however it is simpler for our purposes to begin with a particular construction $\mathbf{T}_{S}$ that forms a reasonable "first approximation" to the free twisted agreeable monoid. The final free algebra is then obtained by taking a quotient of $\mathbf{T}_{S}$.

Let $T_{S}^{0}$ be the set of all expressions of the form

$$
S^{*} \cup\left\{t_{0}\left(s_{1} * t_{1}\right)\left(s_{2} * t_{2}\right) \cdots\left(s_{k} * t_{k}\right) \mid t_{0}, t_{1}, t_{2}, \ldots, t_{k}, s_{1}, s_{2}, \ldots, s_{k} \in S^{*}\right\} .
$$

Note that $T_{S}^{0}$ is not an algebra, merely a set of expressions of the above specified form (having no nested occurrences of $*$ in particular).

It is easily seen that in the variety of twisted agreeable monoids, any expression over $S$ can be re-written to look like an element of $T_{S}^{0}$, by successive use of the laws. For instance, in this variety we have $b *(c * d) \stackrel{13}{\approx}(c * d) * b \approx 1(c * d) * b \underset{16}{\approx}(1 * b)(c * d)$. (Note that this simplification fails for non-monoid examples - we consider the non-monoid case separately.)

Example 3.1. The term $x a(a * b)(a(a * b) * c)[x a(a * b)(a(a * b) * c) * y b(b * c)]$ can be reduced to the agreeable term $x a(a * b)(a * c)(x a * y b)(b * c)$.

Proof. First note that

$$
\begin{aligned}
x a(a * b)(a(a * b) * c) \stackrel{16,15}{ } & x a(a * b)((a * c)(a * b)) \\
& \approx x a(a * b)(a * c)
\end{aligned}
$$

so that $x a(a * b)(a(a * b) * c)[x a(a * b)(a(a * b) * c) * y b(b * c)]$ reduces to $x a(a * b)(a * c)[x a(a * b)(a * c) * y b(b * c)]$. Now, freely using laws 15,13 , we get

$$
\begin{aligned}
x a(a * b)(a * c)[x a(a * b)(a * c) * y b(b * c)] & \stackrel{16}{\approx} x a(a * b)(a * c)[x a * y b(b * c)](a * b)(a * c) \\
& \stackrel{16}{\approx} x a(a * b)(a * c)(x a * y b)(b * c)(a * b)(a * c) \\
& \stackrel{18}{\approx} x a(a * b)(a * c)(x a * y b)(b * c),
\end{aligned}
$$

as required.

The term considered in Example 3.1 is the agreeable term obtained from the left hand side of the Garvac'kiu identity 20 under the term equivalence $a \wedge b \mapsto a(a * b)$. We only claim that there is at least one way to reduce every twisted agreeable term to one in $T_{S}^{0}$, not that this reduction is unique (which fails to be true). Consequently, different elements of $T_{S}^{0}$ may be equal when interpreted in the variety of twisted agreeable semigroups.

Based on the the above examples, we are easily led to a general algorithm. Given an agreeable semigroup term $\phi$ :

- replace any subterm of the form $f *(g * h)$ by one of the form $(1 * f)(g * h)$;

- replace any subterm of the form $(f * g) h$ by one of the form $h(f h * g h)$;

- replace any subterm of the form $(f(g * h) * k)$ by one of the form $(f * k)(g * h)$,

(where $f, g, h, k$ are monoid words). These three replacement rules merely implement laws 15 , 16 and 17 for twisted agreeables. An easy induction argument shows that successive application of these rules must terminate, and the result is necessarily an element of $T_{S}^{0}$ : exactly which element will depend upon the order of application of the three rules above. Elements of $T_{S}^{0}$ with multiple occurrences of the same $(a * b)$ terms are not ruled out by this process.

Before constructing $\mathbf{T}_{S}$, we make some elementary identifications between some of the expressions in $T_{S}^{0}$ (corresponding to axiom (13) and the first identity of (18)). This step could alternatively be built into our final congruence on $\mathbf{T}_{S}$, however this results in a proliferation of trivial steps. We define an equivalence relation $\equiv$ on $T_{S}^{0}$ by $t_{0}\left(s_{1} * t_{1}\right)\left(s_{2} * t_{2}\right) \cdots\left(s_{k} * t_{k}\right) \equiv v_{0}\left(u_{1} * v_{1}\right)\left(u_{2} * v_{2}\right) \cdots\left(u_{k} * v_{k}\right)$ 
if and only if $t_{0}=v_{0}$ and each $\left(s_{i} * t_{i}\right)$ pairs with exactly one $\left(u_{j} * v_{j}\right)$, in such a way that either $\left(s_{i}, t_{i}\right)=\left(u_{j}, v_{j}\right)$ or $\left(s_{i}, t_{i}\right)=\left(v_{j}, u_{j}\right)$. Thus $\equiv$ identifies elements of $T_{S}^{0}$ obtained from one-another by re-ordering the appearance of the $s_{j} * t_{j}$ terms, or by replacing one or more $s_{j} * t_{j}$ by $t_{j} * s_{j}$. Viewed as agreeable semigroup terms, if elements of $T_{S}^{0}$ are equivalent under $\equiv$, then they are certainly equivalent agreeable semigroup terms. Notationally, we will make no distinction between an element $s$ of $T_{S}^{0}$ and its congruence class $s / \equiv$. In other words, in place of an element $s / \equiv$ of $T_{S}^{0} / \equiv$ we write $s$, but we work modulo $\equiv$. The set $T_{S}^{0} / \equiv$, which we denote by $T_{S}$, will be the underlying set for the algebra $\mathbf{T}_{S}$.

We say $t_{0},\left(s_{1} * t_{1}\right),\left(s_{2} * t_{2}\right), \ldots,\left(s_{k} * t_{k}\right)$ are subterms of $t_{0}\left(s_{1} * t_{1}\right)\left(s_{2} * t_{2}\right) \cdots\left(s_{k} * t_{k}\right) \in T_{S}$, and, consistent with the definition of $T_{S}$, we identify $\left(s_{i} * t_{i}\right)$ with $\left(t_{i} * s_{i}\right)$ for this purpose. Thus $t_{1} * s_{1}$ is a subterm of $t_{0}\left(s_{1} * t_{1}\right)\left(s_{2} * t_{2}\right) \cdots\left(s_{k} * t_{k}\right)$.

We now define a multiplication and an operation $*$ on $T_{S}$. For $t=t_{0}\left(s_{1} * t_{1}\right)\left(s_{2} * t_{2}\right) \cdots\left(s_{k} * t_{k}\right)$ and $r=r_{0}\left(q_{1} * r_{1}\right)\left(q_{2} * r_{2}\right) \cdots\left(q_{l} * r_{l}\right)$ in $T_{S}$, define

$$
t r=t_{0} r_{0}\left(s_{1} r_{0} * t_{1} r_{0}\right)\left(s_{2} r_{0} * t_{2} r_{0}\right) \cdots\left(s_{k} r_{0} * t_{k} r_{0}\right)\left(q_{1} * r_{1}\right)\left(q_{2} * r_{2}\right) \cdots\left(q_{l} * r_{l}\right),
$$

and define

$$
t * r=\left(t_{0} * r_{0}\right)\left(s_{1} * t_{1}\right)\left(s_{2} * t_{2}\right) \cdots\left(s_{k} * t_{k}\right)\left(q_{1} * r_{1}\right)\left(q_{2} * r_{2}\right) \cdots\left(q_{l} * r_{l}\right) .
$$

Both operations are easily seen to be well-defined on $T_{S}$ (that is, if $t^{\prime} \equiv t$ and $r^{\prime} \equiv r$ then $t^{\prime} r^{\prime} \equiv t r$ and $\left.t^{\prime} * r^{\prime} \equiv t * r\right)$. The operation - is associative and so this defines a binary semigroup structure, $\mathbf{T}_{S}:=\left\langle T_{S} ; \cdot, *\right\rangle$. either:

We say $t * t$ is redundant in $r=r_{0}\left(q_{1} * r_{1}\right)\left(q_{2} * r_{2}\right) \cdots\left(q_{l} * r_{l}\right)$ if $t * t=q_{i} * r_{i}$ for some $i$ and

- there is another subterm of the form $q_{j} * r_{j}=s t * u$ for some $s, u \in S^{*}$ and $j \neq i$, or

- $r_{0}=s t$ for some $s \in S^{*}$.

(Note that any twisted agreeable semigroup satisfies $(t * t)(s t * u) \approx s t * u$, and $s t(t * t) \approx s t$.)

Let $s=s_{0}\left(s_{1} * s_{1}^{\prime}\right)\left(s_{2} * s_{2}^{\prime}\right) \cdots\left(s_{k} * s_{k}^{\prime}\right) \in T_{S}$. An elementary operation on $s$ is one of the following operations:

(1) removing or adding redundancies: if $(p q * r)$ is a subterm of $s$, or if $s_{0}=p q$, add/delete the subterm $(q * q)$ to/from $s$;

(2) --replacing: if $s_{0}=p q$ and $(q * r)$ is a subterm of $s$, then replace $s_{0}$ by $p r$; or

(3) *-replacing: if $(p q * r)$ and $(q * t)$ are subterms of $s$, then replace $(p q * r)$ by $(p t * r)$.

(Note that addition and removal of redundancies are inverse, while the definition of $\equiv$ ensures that $*$ is commutative, so that both rules 2 and 3 are self inverse.) Now write $s \approx t$ if $s$ can be transformed into $t$ by some sequence of elementary operations.

Theorem 3.2. The relation $\approx$ is a congruence on $\mathbf{T}_{S}$.

Proof. It is clear enough that $\approx$ is an equivalence relation on $\mathbf{T}_{S}$. We show it respects the binary operations.

Let $u=u_{0}\left(u_{1} * u_{1}^{\prime}\right)\left(u_{2} * u_{2}^{\prime}\right) \cdots\left(u_{l} * u_{l}^{\prime}\right) \in T_{S}$. Suppose $s \approx t$, with $s, t$ separated by one step only.

Suppose the single step is a redundancy removal, without loss of generality occurring in passing from $s=s_{0}\left(s_{1} * s_{1}^{\prime}\right) \cdots\left(s_{k} * s_{k}^{\prime}\right)\left(s_{k+1} * s_{k+1}\right)$ to $t$, with $\left(s_{k+1} * s_{k+1}\right)$ absent from $t$; thus $s_{k+1}$ is the end of $s_{0}$ or of an $s_{i}$ or $s_{i}^{\prime}$ for some $i=1,2, \ldots, k$. Then

$$
\begin{aligned}
s * u & =\left(s_{0} * u_{0}\right)\left(s_{1} * s_{1}^{\prime}\right) \cdots\left(s_{k} * s_{k}^{\prime}\right)\left(s_{k+1} * s_{k+1}\right)\left(u_{1} * u_{1}^{\prime}\right) \cdots \\
& \approx\left(s_{0} * u_{0}\right)\left(s_{1} * s_{1}^{\prime}\right) \cdots\left(s_{k} * s_{k}^{\prime}\right)\left(u_{1} * u_{1}^{\prime}\right) \cdots \quad \text { (redundancy removal) } \\
& =t * u .
\end{aligned}
$$

Similarly, because $*$ is commutative, $u * s \approx u * t$. Now considering $s u$, we find that $\left(s_{i} u_{0} * s_{i}^{\prime} u_{0}\right)$ (or $\left.s_{0} u_{0}\right)$ appears and so $\left(s_{k+1} u_{0} * s_{k+1} u_{0}\right)$ is again redundant. So $s u \approx t u$. On the other hand, in $u s$, we have

$$
u s=u_{0} s_{0}\left(u_{1} s_{0} * u_{1}^{\prime} s_{0}\right) \cdots\left(u_{m} s_{0} * u_{m}^{\prime} s_{0}\right)\left(s_{1} * s_{1}^{\prime}\right) \cdots\left(s_{l} * s_{l}^{\prime}\right)\left(s_{k+1} * s_{k+1}\right) .
$$


Clearly $s_{k+1}$ is still the final part of $s_{i}$ or $s_{i}^{\prime}$ for some $i$ and so $\left(s_{k+1} * s_{k+1}\right)$ is redundant; its removal gives $u t$.

Now suppose the single step is a $*$-replacement. We have

$$
\begin{aligned}
s & =s_{0}\left(a s_{1} * s_{2}\right)\left(s_{1} * s_{3}\right) \cdots, \\
t & =s_{0}\left(a s_{3} * s_{2}\right)\left(s_{1} * s_{3}\right) \cdots .
\end{aligned}
$$

Then

$$
\begin{aligned}
s * u & =\left(s_{0} * u_{0}\right)\left(a s_{1} * s_{2}\right)\left(s_{1} * s_{3}\right) \cdots \\
& \approx\left(s_{0} * u_{0}\right)\left(a s_{3} * s_{2}\right)\left(s_{1} * s_{3}\right) \cdots \quad \text { (*-replacement) } \\
& =t * u .
\end{aligned}
$$

Again, commutativity ensures that $u * s \approx u * t$. But we also have that

$$
s u=s_{0} u_{0}\left(a s_{1} u_{0} * s_{2} u_{0}\right)\left(s_{1} u_{0} * s_{3} u_{0}\right) \cdots,
$$

while

$$
\begin{aligned}
t u & =s_{0} u_{0}\left(a s_{3} u_{0} * s_{2} u_{0}\right)\left(s_{1} u_{0} * s_{3} u_{0}\right) \cdots \\
& \left.\approx s_{0} u_{0}\left(a s_{1} u_{0} * s_{2} u_{0}\right)\left(s_{1} u_{0} * s_{3} u_{0}\right) \cdots \quad \text { (*-replacement }\right) \\
& =s u .
\end{aligned}
$$

The proof that $u s \approx u t$ is similarly routine.

Now we consider -replacement. Without loss of generality we have that

$$
\begin{aligned}
s & =s_{0}^{\prime} s_{0}\left(s_{0} * s_{1}\right) \cdots \\
t & =s_{0}^{\prime} s_{1}\left(s_{0} * s_{1}\right) \cdots .
\end{aligned}
$$

Then

$$
\begin{aligned}
t * u & =\left(s_{0}^{\prime} s_{0} * u_{0}\right)\left(s_{0} * s_{1}\right) \cdots \\
& \left.\approx\left(s_{0}^{\prime} s_{1} * u_{0}\right)\left(s_{0} * s_{1}\right) \cdots \quad \text { (*-replacement }\right) \\
& =s * u
\end{aligned}
$$

Again, commutativity ensures that $u * s \approx u * t$. For the multiplicative case (with $s, t$ and $u$ as before) we have

$$
\begin{aligned}
u s & =u_{0} s_{0}^{\prime} s_{0}\left(u_{1} s_{0}^{\prime} s_{0} * u_{1}^{\prime} s_{0}^{\prime} s_{0}\right) \cdots\left(s_{0} * s_{1}\right) \cdots \\
& \approx u_{0} s_{0}^{\prime} s_{1}\left(u_{1} s_{0}^{\prime} s_{1} * u_{1}^{\prime} s_{0}^{\prime} s_{1}\right) \cdots \quad \text { (-replacement and many *-replacements) } \\
& =u t .
\end{aligned}
$$

The proof that $s u \approx t u$ is even easier.

Thus providing $t$ is obtained from $s$ by a single elementary operation, we have that $s * u \approx t * u$, $s u \approx t u$ and $u s \approx u t$. In the general case, where a sequence of elementary operations is required to transform $s$ into $t$, we may use a simple induction argument on the length of this sequence to again obtain $s * u \approx t * u, s u \approx t u$ and $u s \approx u t$. Hence $\approx$ is a congruence on $\mathbf{T}_{S}$. following

We have now shown that $\mathbf{T}_{S} / \approx$ is a well-defined algebraic object. It remains to prove the

Theorem 3.3. $\mathbf{T}_{S} / \approx$ is a twisted agreeable monoid, with agreeable operation $*$.

Proof. Certainly $\mathbf{T}_{S} / \approx$ is a monoid since $\mathbf{T}_{S}$ was already. We now verify laws $12-17$ of Section 2 . Note that for purely semigroup terms $c, d, *$-replacement and redundancy imply that

$$
(c * d)(c * d) \approx(c * c)(c * d) \approx(c * d) .
$$

Now let $s=s_{0}\left(s_{1} * s_{1}^{\prime}\right)\left(s_{2} * s_{2}^{\prime}\right) \cdots\left(s_{k} * s_{k}^{\prime}\right)$. Then

$$
\begin{aligned}
(s * s) & =\left(s_{0} * s_{0}\right)\left(s_{1} * s_{1}^{\prime}\right)\left(s_{2} * s_{2}^{\prime}\right) \cdots\left(s_{k} * s_{k}^{\prime}\right)\left(s_{1} * s_{1}^{\prime}\right)\left(s_{2} * s_{2}^{\prime}\right) \cdots\left(s_{k} * s_{k}^{\prime}\right) \\
& \approx\left(s_{0} * s_{0}\right)\left(s_{1} * s_{1}^{\prime}\right)\left(s_{2} * s_{2}^{\prime}\right) \cdots\left(s_{k} * s_{k}^{\prime}\right) .
\end{aligned}
$$


So

$$
\begin{aligned}
s[s * s] & \approx s_{0}\left(s_{1} * s_{1}^{\prime}\right)\left(s_{2} * s_{2}^{\prime}\right) \cdots\left(s_{k} * s_{k}^{\prime}\right)\left[\left(s_{0} * s_{0}\right)\left(s_{1} * s_{1}^{\prime}\right)\left(s_{2} * s_{2}^{\prime}\right) \cdots\left(s_{k} * s_{k}^{\prime}\right)\right] \\
& \approx s_{0}\left(s_{0} * s_{0}\right)\left(s_{1} * s_{1}^{\prime}\right)\left(s_{2} * s_{2}^{\prime}\right) \cdots\left(s_{k} * s_{k}^{\prime}\right) \\
& \approx s_{0}\left(s_{1} * s_{1}^{\prime}\right)\left(s_{2} * s_{2}^{\prime}\right) \cdots\left(s_{k} * s_{k}^{\prime}\right) \\
& =s
\end{aligned}
$$

showing that axiom 12 is satisfied.

The identity $a * b \approx b * a$ (axiom 13) is already satisfied by $\mathbf{T}_{S}$ (whence $\mathbf{T}_{S} / \approx$ ) because for any $s, t \in T_{S}^{0}$ we have $s * t \equiv t * s$.

Let $s$ be as above and let $t=t_{0}\left(t_{1} * t_{1}^{\prime}\right)\left(t_{2} * t_{2}^{\prime}\right) \cdots\left(t_{r} * t_{r}^{\prime}\right)$. Then

$$
\begin{aligned}
s[s * t]= & s_{0}\left(s_{1} * s_{1}^{\prime}\right)\left(s_{2} * s_{2}^{\prime}\right) \cdots\left(s_{k} * s_{k}^{\prime}\right)\left[\left(s_{0} * t_{0}\right)\left(s_{1} * s_{1}^{\prime}\right)\left(s_{2} * s_{2}^{\prime}\right) \cdots\right. \\
& \left.\left(s_{k} * s_{k}^{\prime}\right) \cdot\left(t_{1} * t_{1}^{\prime}\right)\left(t_{2} * t_{2}^{\prime}\right) \cdots\left(t_{r} * t_{r}^{\prime}\right)\right] \\
\approx & s_{0}\left(s_{0} * t_{0}\right)\left(s_{1} * s_{1}^{\prime}\right)\left(s_{2} * s_{2}^{\prime}\right) \cdots\left(s_{k} * s_{k}^{\prime}\right)\left(t_{1} * t_{1}^{\prime}\right)\left(t_{2} * t_{2}^{\prime}\right) \cdots\left(t_{r} * t_{r}^{\prime}\right) \\
\approx & t_{0}\left(s_{0} * t_{0}\right)\left(s_{1} * s_{1}^{\prime}\right)\left(s_{2} * s_{2}^{\prime}\right) \cdots\left(s_{k} * s_{k}^{\prime}\right)\left(t_{1} * t_{1}^{\prime}\right)\left(t_{2} * t_{2}^{\prime}\right) \cdots\left(t_{r} * t_{r}^{\prime}\right),
\end{aligned}
$$

by $(\mathcal{Q})$ and --replacement. By symmetry this must also be equivalent modulo $\approx$ to $t[s * t]$, showing that axiom 14 holds. of $*$ on $\mathbf{T}_{S}$.

The identity $(a * b) *(c * d) \approx(a * b)(c * d)$ (axiom 15) holds immediately from our definition

We next show that $(a * b) *(c * d) \approx(a * b)(c * d)$ (axiom 16) holds. Let $s, t$ be as above and w.l.o.g. assume $u * v=p_{1} p_{2} \cdots p_{m}$ (where each $\left.p_{i}=\left(u_{i} * v_{i}\right), i=1,2, \ldots, m\right)$. Then

$$
s(u * v)=s_{0}\left(s_{1} * s_{1}^{\prime}\right)\left(s_{2} * s_{2}^{\prime}\right) \cdots\left(s_{k} * s_{k}^{\prime}\right) p_{1} p_{2} \cdots p_{m},
$$

so

$$
\begin{aligned}
{[s(u * v)] * t } & =\left(s_{0} * t_{0}\right)\left(s_{1} * s_{1}^{\prime}\right) \cdots\left(s_{k} * s_{k}^{\prime}\right) p_{1} p_{2} \cdots p_{m}\left(t_{1} * t_{1}^{\prime}\right) \cdots\left(t_{r} * t_{r}^{\prime}\right) \\
& =\left[\left(s_{0} * t_{0}\right)\left(s_{1} * s_{1}^{\prime}\right) \cdots\left(s_{k} * s_{k}^{\prime}\right)\left(t_{1} * t_{1}^{\prime}\right) \cdots\left(t_{r} * t_{r}^{\prime}\right)\right]\left[p_{1} p_{2} \cdots p_{m}\right] \\
& =(s * t)(u * v) .
\end{aligned}
$$

(This law again holds in $\mathbf{T}_{S}$.)

Finally, we show $(a * b) c=c(a c * b c)$ (axiom 17). Let $s, t$ be as above and let $u=u_{0}\left(u_{1} *\right.$ $\left.u_{1}^{\prime}\right)\left(u_{2} * u_{2}^{\prime}\right) \cdots\left(u_{p} * u_{p}^{\prime}\right)$. Then

$$
\begin{aligned}
{[s * t] u=\left[\left(s_{0} * t_{0}\right)\left(s_{1} * s_{1}^{\prime}\right) \cdots\left(s_{k} * s_{k}^{\prime}\right)\left(t_{1} * t_{1}^{\prime}\right) \cdots\left(t_{r} * t_{r}^{\prime}\right)\right] } & \cdot u_{0}\left(u_{1} * u_{1}^{\prime}\right)\left(u_{2} * u_{2}^{\prime}\right) \cdots\left(u_{p} * u_{p}^{\prime}\right) \\
=u_{0}\left(s_{0} u_{0} * t_{0} u_{0}\right)\left(s_{1} u_{0} *\right. & \left.s_{1}^{\prime} u_{0}\right) \cdots\left(s_{k} u_{0} * s_{k}^{\prime} u_{0}\right) \\
& \cdot\left(t_{1} u_{0} * t_{1}^{\prime} u_{0}\right) \cdots\left(t_{r} u_{0} * t_{r}^{\prime} u_{0}\right)\left(u_{1} * u_{1}^{\prime}\right) \cdots\left(u_{p} * u_{p}^{\prime}\right)
\end{aligned}
$$

whereas

$$
\begin{aligned}
& u[s u * t u]=u_{0}\left(u_{1} * u_{1}^{\prime}\right) \cdots\left(u_{p} * u_{p}^{\prime}\right) {\left[\left(s_{0} u_{0} * t_{0} u_{0}\right)\left(s_{1} u_{0} * s_{1}^{\prime} u_{0}\right) \cdots\left(s_{k} u_{0} * s_{k}^{\prime} u_{0}\right)\right.} \\
&\left.\cdot\left(u_{1} * u_{1}^{\prime}\right) \cdots\left(u_{p} * u_{p}^{\prime}\right)\left(t_{1} u_{0} * t_{1}^{\prime} u_{0}\right) \cdots\left(t_{r} u_{0} * t_{r}^{\prime} u_{0}\right)\right] .
\end{aligned}
$$

Then $u[s u * t u] \approx[s * t] u$ as required. The result now follows.

Corollary 3.4. $\mathbf{T}_{S} / \approx$ is the free twisted agreeable monoid on generators $S$.

Proof. This is essentially because we only used the laws of twisted agreeable monoids to define $\mathbf{T}_{S}$ and $\mathbf{T}_{S} / \approx$.

It remains to give a canonical simplifier, that is, an algorithm which, when provided with an element $a$ of $\mathbf{T}_{S}$, produces another element $N(a) \in a / \approx$ and such that $a \approx b$ implies $N(a)=N(b)$. This will obviously provide a solution to the word problem on the free twisted agreeable monoid (equivalently, the equational problem for the variety of twisted agreeable monoids). 


\section{LEFT COMPATIBLE ORDERS AND RIGHT REDUCTION FOR SEMIGROUP WORDS.}

Our canonical simplifier $N$ will be defined relative to a particular fixed left compatible order $\leq$ on elements of $S^{*}$, which is a total order satisfying

- there is no infinite descending chain $s_{1}>s_{2}>s_{3}>\cdots$, and

- $s<t \Rightarrow u s<u t$ for all $s, t, u \in S^{*}$.

Examples of such orders abound of course. We assume we have a fixed one in what follows, say $\leq$.

In order to carry the analysis further, we need to consider the notion of (right) reduction for $S^{*}$. Given an ordered pair $(u, v)$ of elements of $S^{*}$ with $u>v$, we say $(u, v)$ reduces $s$ to $t$ if $s=k u$ and $t=k v$ for some $k \in S^{*}$. The idea is that we "replace $u$ on the right of $s$ by $v$ to give $t$ ". Note that if $s$ reduces to $t$, then $s>t$.

Given a finite subset

$$
F=\left\{\left(u_{1}, v_{1}\right),\left(u_{2}, v_{2}\right), \ldots,\left(u_{k}, v_{k}\right)\right\}
$$

of $S^{*} \times S^{*}$ with $u_{i}>v_{i}$ for each $i$, there is an induced reduction relation $\rightarrow_{F}$ on $S^{*}$, defined by setting $s \rightarrow_{F} t$ if there is $(u, v) \in F$ which reduces $s$ to $t$. We call $F$ a reduction set, or an $R$-set. We call $u$ the head and $v$ the tail of $(u, v) \in F$.

The following is immediate from the definition of a compatible order and the fact that reduction makes words smaller.

Proposition 4.1. For $F$ an $R$-set in $S^{*} \times S^{*}, \rightarrow_{F}$ is a Noetherian reduction relation; that is, there is no infinite sequence of reductions $s \rightarrow_{F} s_{1} \rightarrow_{F} s_{2} \rightarrow_{F} \cdots$.

Let $\rightarrow_{F}^{*}$ be the reflexive transitive closure of $\rightarrow_{F}$, let $\leftrightarrow_{F}$ denote its reflexive symmetric closure, and let $\leftrightarrow_{F}^{*}$ denote its reflexive symmetric transitive closure (that is, the equivalence relation generated by $\rightarrow_{F}$ ). The next result proves quite useful.

Lemma 4.2. Suppose $F$ is an $R$-set. If $s, t \in S^{*}$ are such that $s \leftrightarrow_{F}^{*} t$, then $k s \leftrightarrow{ }_{F}^{*} k t$ for any $k \in S^{*}$

Proof. First suppose $s \rightarrow_{F} t$. Then $s=r u$ and $t=r v$ where $(u, v) \in F$. So $k s=k r u$ and $k t=k r v$, and so $k s \rightarrow_{F} k t$ also. The more general result for terms in $S^{*}$ linked by arbitrarily many uses of $\leftrightarrow_{F}$ now follows easily by induction.

We say the reduction set $F$ is mutually reduced if no head or tail of any pair in $F$ can be reduced by any other pair in $F$.

Lemma 4.3. Let $F$ be a mutually reduced $R$-set. Then for $s \in S^{*}$, there is at most one pair in $F$ that can reduce $s$.

Proof. Suppose $(t, u) \in F$ and $s$ can be reduced using $(t, u)$. Thus $s=k t$ for some $k \in S^{*}$. If also $s$ can be reduced by some other $(u, v) \in F$, then $s=k^{\prime} u$ for some $k^{\prime} \in S^{*}$, so that either $t=r u$ or $u=r t$ for some $r \in S^{*}$. But this would mean $F$ was not mutually reduced, a contradiction.

It therefore follows that if $F$ is mutually reduced, $\rightarrow_{F}$ is at most single-valued when applied to elements of $S^{*}$.

Given any reduction relation, one can discuss normal forms. In this case, if $F$ is an R-set, we say $s \in S^{*}$ is in normal form relative to $\rightarrow_{F}$ if no pair in $F$ reduces $s$.

Corollary 4.4. Let $F$ be a mutually reduced $R$-set. If $s, t$ are normal forms relative to $\rightarrow_{F}$, and if $s \leftrightarrow{ }_{F}^{*} t$, then $s=t$.

Proof. Since $\rightarrow_{F}$ is trivially confluent (at most one reduction is ever possible by Lemma 4.3), then by a result in [3], if $s \leftrightarrow_{F}^{*} t$ then there exists a (unique) normal form $u$ for which $s \rightarrow_{F}^{*} u$ and $t \rightarrow_{F}^{*} u$. Since $s, t$ are assumed to be in normal form, we must have $s=t=u$.

There is an obvious algorithm for finding the normal form $N_{F}(s)$ of any $s \in S^{*}$ relative to a mutually reduced R-set $F$ : simply reduce $s$ repeatedly using $F$ until this is no longer possible. Moreover, $N_{F}(s) \leftrightarrow_{F}^{*} s$ and $N_{F}(s)$ is the unique normal form with this property by the above corollary. 
We now give a method for converting any R-set $F$ into a mutually reduced $G$ for which $\leftrightarrow_{F}^{*}=\leftrightarrow_{G}^{*}$

Given R-sets $F$ and $G$, we say $F$ transforms by replacement to $G$ if there exists $(s, t) \in F$ for which either:

(R1) $G=F \backslash\{(s, s)\}$, or

(R2) $G=F \backslash\{(s, t)\} \cup\left\{\left(s^{\prime}, t^{\prime}\right)\right\}$, where either:

(a) $s$ reduces to $s^{\prime}$ using $(u, v) \in F \backslash\{(s, t)\}$ with $s^{\prime} \geq t$ and $t^{\prime}=t$; or

(b) $s$ reduces to $t^{\prime}$ using $(u, v) \in F \backslash\{(s, t)\}$ with $t^{\prime}<t$ and $s^{\prime}=t$; or

(c) $t$ reduces to $t^{\prime}$ using $(u, v) \in F \backslash\{(s, t)\}$ and $s^{\prime}=s$.

In summary, $F$ transforms by replacement to $G$ if one of the pairs $(s, t)$ in $F$ can have either its head or tail reduced by the rest of $F$ and then $G$ is the R-set obtained by replacing $(s, t)$ by the pair $\left(s^{\prime}, t^{\prime}\right)$ that $(s, t)$ reduces to (adjusting the ordering of $\left(s^{\prime}, t^{\prime}\right)$ if needed, or eliminating it altogether if $s^{\prime}=t^{\prime}$ become equal).

Lemma 4.5. Suppose $F$ transforms by replacement to $G$. Then $\leftrightarrow_{F}^{*}=\leftrightarrow_{G}^{*}$.

Proof. First suppose that $a \rightarrow_{F} b$. Thus there is $(s, t) \in F$ which reduces $a$ to $b$, so $a=m s$ and $b=m t$ for some $m \in S^{*}$.

Obviously if $(s, t) \in G$, there is nothing to show. If $(s, t) \notin G$, then there will be $(u, v) \in$ $F \backslash\{(s, t)\}$ which reduces either $s$ or $t$, and there are several cases to consider.

(R1): Suppose $G=F \backslash\{(s, s)\}$. It is then immediate that $a \rightarrow_{G} b$ since the presence or absence of elements of the form $(s, s)$ does not affect $\rightarrow_{F}$, so $\rightarrow_{G}=\rightarrow_{F}$.

Suppose $G=F \backslash\{(s, t)\} \cup\left\{\left(s^{\prime}, t^{\prime}\right)\right\}$ where $s^{\prime}, t^{\prime}$ are as in the definition above. We treat each case separately.

(R2a): If $s$ reduces to $s^{\prime}$ using $(u, v)$ with $s^{\prime} \geq t$ and $t^{\prime}=t$, then $s=k u$ and $s^{\prime}=k v \geq t$ for some $k \in S^{*}$, so $a=m s=m k u \rightarrow_{G} m k v=m s^{\prime} \rightarrow_{G} m t^{\prime}=m t=b$.

(R2b): If $s$ reduces to $t^{\prime}$ using $(u, v)$ with $t^{\prime}<t$ and $s^{\prime}=t$, then $s=k u$ and $t^{\prime}=k v<t$ for some $k \in S^{*}$, so $a=m s=m k u \rightarrow_{G} m k v=m t^{\prime}$, while $b=m t=m s^{\prime} \rightarrow_{G} m t^{\prime}$, so $a \leftrightarrow_{G}^{*} b$.

(R2c): If $t$ reduces to $t^{\prime}$ using $(u, v)$ and $s^{\prime}=s$, then $t=k u$ and $t^{\prime}=k v$ for some $k \in S^{*}$, so $a=m s=m s^{\prime} \rightarrow_{G} m t^{\prime}=m k v$, while also $b=m t=m k u \rightarrow_{G} m k v$, so $a \leftrightarrow_{G}^{*} b$.

We have now shown that if $a \rightarrow_{F} b$ then $a \leftrightarrow_{G}^{*} b$. An obvious induction argument then establishes that if $a \leftrightarrow_{F}^{*} b$, then $a \leftrightarrow_{G}^{*} b$.

Conversely, suppose that $a \rightarrow_{G} b$. Again, the only interest is if $a$ reduces to $b$ using $\left(s^{\prime}, t^{\prime}\right) \in G$ with $\left(s^{\prime}, t^{\prime}\right) \notin F$, in which case $a=m s^{\prime}$ and $b=m t^{\prime}$ for some $m \in S^{*}$. Moreover, there is $(s, t) \in F$ which is not in $G$, and $\left(s^{\prime}, t^{\prime}\right)$ could only have arisen in one of the above ways from $(s, t)$; we examine these in turn.

(R2a) If $s$ reduces to $s^{\prime}$ using $(u, v) \in F \cap G$ with $s^{\prime} \geq t$ and $t^{\prime}=t$, then again $s=k u$ and $s^{\prime}=k v \geq t$ for some $k$, so $m k u \rightarrow_{F} m k v=m s^{\prime}=a$, while $m k u=m s \rightarrow_{F} m t=m t^{\prime}=b$, so $a \leftrightarrow{ }_{F}^{*} b$.

(R2b) If $s$ reduces to $t^{\prime}$ using $(u, v)$ with $t^{\prime}<t$ and $s^{\prime}=t$, then again $s=k u$ and $t^{\prime}=k v<t$ for some $k \in S^{*}$, so $m s \rightarrow_{F} m t=m s^{\prime}=a$, while also $m s=m k u \rightarrow_{F} m k v=m t^{\prime}=b$, so $a \leftrightarrow{ }_{F}^{*} b$.

(R2c) Finally, if $t$ reduces to $t^{\prime}$ using $(u, v)$ and $s^{\prime}=s$, then again $t=k u$ and $t^{\prime}=k v$ for some $k \in S^{*}$, so $a=m s^{\prime}=m s \rightarrow_{F} m t=m k u \rightarrow_{F} m k v=m t^{\prime}=b$, and then $a \leftrightarrow_{F}^{*} b$.

Again, induction establishes that if $a \leftrightarrow_{G}^{*} b$, then $a \leftrightarrow_{F}^{*} b$. Combining this with the above, we have shown that $\leftrightarrow_{F}^{*}=\leftrightarrow_{G}^{*}$.

Lemma 4.6. Suppose $F, G$ are mutually reduced $R$-sets for which the equivalence relations $\leftrightarrow_{F}^{*}$ and $\leftrightarrow_{G}^{*}$ are equal. Then $F=G$.

Proof. Suppose

$$
F=\left\{\left(a_{1}, b_{1}\right),\left(a_{2}, b_{2}\right), \ldots,\left(a_{n}, b_{n}\right)\right\}, G=\left\{\left(c_{1}, d_{1}\right),\left(c_{2}, d_{2}\right), \ldots,\left(c_{m}, d_{m}\right)\right\} .
$$


Let $N_{F}$ and $N_{G}$ be the associated normal form functions. Now $c_{1} \rightarrow_{G} d_{1}$ trivially, so $c_{1} \leftrightarrow \leftrightarrow_{F}^{*} d_{1}$, and so $N_{F}\left(c_{1}\right)=N_{F}\left(d_{1}\right)$ by Corollary 4.4 , and since $N_{F}(s) \leq s$ for any $s \in S^{*}$, it follows that $c_{1}$ must be reducible by some pair in $F$; say $c_{1}=d a_{i}$ for some $d \in S^{*}$ and $i$. Similarly, $a_{i}=e c_{j}$ for some $e$ and $j$. So $c_{1}=d e c_{j}$, a contradiction unless $d=e=1$, in which case $j=1$ and $a_{i}=c_{1}$. So $c_{1}$ is the head of a pair in $F$. Repeating this for each head in $G$ we find that every head in $G$ is a head in $F$, and then reversing the argument shows that the pairs in $F, G$ have identical heads (and in particular $m=n)$. So without loss of generality we can write $F=\left\{\left(a_{1}, b_{1}\right),\left(a_{2}, b_{2}\right), \ldots,\left(a_{n}, b_{n}\right)\right\}$ and $G=\left\{\left(a_{1}, d_{1}\right),\left(a_{2}, d_{2}\right), \ldots,\left(a_{n}, d_{n}\right)\right\}$. Now if $d_{1}$ is not in normal form relative to $F$, then $d_{1}=k a_{j}$ for some $k \in S^{*}$ and some $j$, a contradiction to $G$ being mutually reduced, so $d_{1}$ must be in normal form relative to $F$. But $a_{1} \leftrightarrow_{F}^{*} d_{1}$, so $N_{F}\left(a_{1}\right)=d_{1}$. But it is clear that $N_{F}\left(a_{1}\right)=b_{1}$, so $b_{1}=d_{1}$; similarly $b_{i}=d_{i}$ for all $i$, and so $F=G$.

Theorem 4.7. For a given $R$-set $F \subseteq S^{*} \times S^{*}$, there is a unique mutually reduced $R$-set $G$ for which the equivalence relations $\leftrightarrow_{F}^{*}$ and $\leftrightarrow_{G}^{*}$ are equal.

Proof. Suppose $F_{0}=F=\left\{\left(u_{1}, v_{1}\right),\left(u_{2}, v_{2}\right), \ldots,\left(u_{k}, v_{k}\right)\right\}$ is an R-set. Choose $\left(u_{i}, v_{i}\right) \in F$ such that $u_{i}$ or $v_{i}$ can be reduced using $\left(u_{j}, v_{j}\right)$ for some $j \neq i$; thus $F$ transforms by replacement to $F_{1}$ say. Repeat with $F_{1}$ to give $F_{2}$, and so on. As an $R$-set is finite, eventually, no further transformations by replacement will be possible since the number of pairs $(u, v)$ is not increasing and one element in some pair is being made smaller in each iteration. The final R-set in the chain, say $F_{n}$, will thus be mutually reduced. By Lemma $4.5, \leftrightarrow{ }_{F}^{*}=\leftrightarrow_{F_{1}}^{*}=\cdots=\leftrightarrow_{F_{n}}^{*}$, so $\leftrightarrow_{F}^{*}=\leftrightarrow_{F_{n}}^{*}$. By Lemma $4.6, F_{n}$ is the unique mutually reduced $\mathrm{R}$-set with this property.

Any R-set can be converted to its unique equivalent mutually reduced R-set by the method described in the last proof.

Example 4.8. Consider the usual lexicographic ordering over the free monoid $\{a, b, c, x, y\}^{*}$. Then $R$-set $\{(b, a),(c, a),(y b, x a),(c, b)\}$ reduces to the mutually reduced $R$-set $\{(b, a),(c, a),(y a, x a)\}$.

Proof. Using the reduction rules we get:

$$
\begin{aligned}
\{(b, a),(c, a),(y b, x a),(c, b)\} & \stackrel{\mathrm{R} 2 c}{\rightarrow}\{(b, a),(c, a),(y b, x a),(c, a)\} \\
& \stackrel{\mathrm{R} 2 \mathrm{a}}{\rightarrow}\{(b, a),(c, a),(y b, x a),(a, a)\} \\
& \stackrel{\mathrm{R} 1}{\rightarrow}\{(b, a),(c, a),(y b, x a)\} \\
& \stackrel{\mathrm{R} 2 \mathrm{a}}{\rightarrow}\{(b, a),(c, a),(y a, x a)\} .
\end{aligned}
$$

Recall that the uniform word problem for monoids (which is undecidable) is equivalent to the membership problem for finitely generated congruences on free monoids. Specifically, if $\langle A: R\rangle$ is a finite presentation and $u, v$ is a pair of words in the alphabet of $A$, then $u=v$ in $\langle A: R\rangle$ if and only if $(u, v)$ is contained in the congruence on $A^{*}$ generated by the pairs $\{(s, t): s=t \in R\}$. In this section we have essentially shown that the corresponding problem for finitely generated left congruences is decidable.

Lemma 4.9. Let $A$ be a finite alphabet, $\leq$ a left compatible order on $A^{*}$ and $R=\left\{\left(u_{1}, v_{1}\right), \ldots,\left(u_{n}, v_{n}\right)\right\}$ a finite subset of $A^{*} \times A^{*}$ with $u_{i} \geq v_{i}$ for $i=1, \ldots, n$. Then $\leftrightarrow_{R}^{*}$ is the smallest left congruence containing $R$.

Proof. Clearly $R \subseteq \leftrightarrow_{R}^{*}$. Now $\leftrightarrow_{R}^{*}$ is itself a left congruence since $u \leftrightarrow{ }_{R}^{*} v$ implies $w u \leftrightarrow_{R}^{*} w v$ for any $u, v, w \in A^{*}$. Now let $\theta$ be any left congruence containing $R$. For any word $w$ and pair $\left(u_{i}, v_{i}\right) \in R$, we have $\left(w u_{i}, w v_{i}\right) \in \theta$ and hence $\rightarrow{ }_{R} \subseteq \theta$. But then $\leftrightarrow_{R}^{*} \subseteq \theta$, because $\theta$ is an equivalence containing $\rightarrow_{R}$, while $\leftrightarrow_{R}^{*}$ is the smallest equivalence containing $\rightarrow_{R}$. Hence $\leftrightarrow_{R}^{*}$ is the smallest left congruence containing $R$. 
Corollary 4.10. There is an algorithm to solve the following decision problem: given a finite alphabet $A$, a finite subset $R \subseteq A^{*} \times A^{*}$ and a pair $(u, v) \in A^{*} \times A^{*}$, decide if $(u, v)$ is contained in the left congruence generated by $R$.

\section{The twisted Agreeable Semigroup Equational PROBlem SOlVEd.}

Given a compatible order on $S^{*}$, we say that $s \in T_{S}$ is written in standard form (relative to this ordering) when it is written as

$$
s=s_{0}\left(s_{1} * s_{1}\right)\left(s_{2} * s_{2}\right) \cdots\left(s_{k} * s_{k}\right)\left(u_{1} * v_{1}\right)\left(u_{2} * v_{2}\right) \cdots\left(u_{r} * v_{r}\right),
$$

where $u_{i}>v_{i}$ for all $i$. (The definition of $\equiv$ ensures that every element of $T_{S}$ can be represented in this way.) Then let $F_{s}=\left\{\left(u_{1}, v_{1}\right),\left(u_{2}, v_{2}\right), \ldots,\left(u_{k}, v_{k}\right)\right\}$, an R-set. Obviously $F_{s}$ is completely determined by $s$, and is not dependent on the way it is written in standard form.

Example 5.1. Relative to the usual lexicographic ordering on $\{a, b, c, x, y\}^{*}$, the equivalent standard form for the term $x a(a * b)(a * c)(x a * y b)(b * c)$ of Example 3.1 is $x a(b * a)(c * a)(c * b)(y b * x a)$. The corresponding $R$-set is the R-set $\{(b, a),(c, a),(c, b),(y b, x a)\}$ of Example 4.8.

We say the standard form $s$ as above is irreducible if

- none of the $\left(s_{i} * s_{i}\right)$ is redundant in $s$, and

- no $u_{i}$ is a final segment of any $s_{j}, u_{\ell}$ or $v_{\ell}(0 \leq j \leq k, 0 \leq \ell \leq r$, and $\ell \neq i)$.

The first condition simply says that no redundancies can be eliminated, and in particular that no $\left(s_{i} * s_{i}\right)$ term occurs twice. The second condition says that neither $*$-replacement nor -replacement using the term $u_{i} * v_{i}$ is possible, if $u_{i}$ is to be replaced by $v_{i}$. This means that replacements of either kind can never reduce the size of the various semigroup words occurring in $s$.

The following is immediate from the definition of irreducibility.

Lemma 5.2. If $s \in T_{S}$ is irreducible, then $F_{s}$ is mutually reduced.

Lemma 5.3. If $s=s_{0}\left(s_{1} * s_{1}\right)\left(s_{2} * s_{2}\right) \cdots\left(s_{k} * s_{k}\right)\left(u_{1} * v_{1}\right)\left(u_{2} * v_{2}\right) \cdots\left(u_{r} * v_{r}\right)$ is irreducible, then for any $w \in S^{*}, N_{F_{s}}\left(w s_{i}\right)=w s_{i}$ for $i=1,2, \ldots, r$.

Proof. If, without loss of generality, $N_{F_{s}}\left(w s_{1}\right) \neq w s_{1}$, then $w s_{1}$ can be reduced using $F_{s}$, so there exists $w^{\prime}, w^{\prime \prime} \in S^{*}$ such that $w=w^{\prime} w^{\prime \prime}$ and $\left(w^{\prime \prime} s, g\right) \in F_{s}$ with $w^{\prime \prime} s_{1}>g \in S^{*}$. Hence $\left(w^{\prime \prime} s_{1} * g\right)$ occurs as one of the $\left(u_{j} * v_{j}\right)$ subterms of $s$ and so $\left(s_{1} * s_{1}\right)$ is redundant, a contradiction to $s$ being irreducible.

Lemma 5.4. If $s, t \in T_{S}$ and $s \approx t$, then $\leftrightarrow_{F_{s}}^{*}=\leftrightarrow_{F_{t}}^{*}$.

Proof. If $s$ transforms to $t$ by removing or adding a redundancy, obviously $F_{s}=F_{t}$ since the $u_{i} * v_{i}$ terms are not altered; the same goes for --replacing.

Suppose $s$ transforms to $t$ by a $*$-replacement, so that $(p q * r)$ in $s$ becomes $(p u * r)$ in $t$, by virtue of the fact that $(q * u)$ occurs in $s$. Then $(q * u)$ will occur in $t$ also. Hence if $q>u$, then $F_{s}$ transforms by replacement to $F_{t}$, while if $u>q$, then $F_{t}$ transforms by replacement to $F_{s}$; either way, $\leftrightarrow_{F_{s}}^{*}=\leftrightarrow_{F_{t}}^{*}$ by Lemma 4.5 .

Hence if $s$ transforms to $t$ by some elementary operation, then $\leftrightarrow_{F_{s}}^{*}=\leftrightarrow_{F_{t}}^{*}$. An easy induction on the number of elementary operations needed to transform $s$ to $t$ when $s \approx t$ establishes that $\leftrightarrow_{F_{s}}^{*}=\leftrightarrow_{F_{t}}^{*}$ in this case also.

Corollary 5.5. If $s, t \in T_{S}$ are both irreducible and $s \approx t$, then $F_{s}=F_{t}$.

Proof. Certainly $\leftrightarrow_{F_{s}}^{*}=\leftrightarrow_{F_{t}}^{*}$ by the previous lemma, and moreover both $F_{s}$ and $F_{t}$ are mutually reduced by Lemma 5.2 , so by Lemma $4.6, F_{s}=F_{t}$.

Lemma 5.6. Let $s \in T_{S}$ have a subterm $(a * b)$, for some $a, b \in S^{*}$. If a sequence of elementary transformations of $s$ alters this subterm to become $(c * d)$, then $a \leftrightarrow{ }_{F_{s}}^{*} c$ and $b \leftrightarrow{ }_{F_{s}}^{*} d$. 
Proof. Suppose $(u v * w)$ becomes $\left(u v^{\prime} * w\right)$ after an elementary transformation of $s$. Then clearly $\left(v * v^{\prime}\right)$ must occur in $s$, so one of $\left(v, v^{\prime}\right)$ or $\left(v^{\prime}, v\right)$ occurs in $F_{s}$. So $v \leftrightarrow F_{s}^{*} v^{\prime}$, and then $u v \leftrightarrow{ }_{F_{s}}^{*} u v^{\prime}$ by Lemma 4.2. A simple inductive argument extends this argument to cases where more than one elementary transformation is needed.

Theorem 5.7. If $s, t \in T_{S}$ are irreducible relative to some fixed compatible ordering of $S^{*}$ and $s \approx t$, then $s=t$.

Proof. It is immediate from Corollary 5.5 that if two irreducible terms $s, t \in T_{S}$ are $\approx$-related then we must have

$$
\begin{aligned}
& s=s_{0}\left(s_{1} * s_{1}\right)\left(s_{2} * s_{2}\right) \cdots\left(s_{k} * s_{k}\right)\left(u_{1} * v_{1}\right)\left(u_{2} * v_{2}\right) \cdots\left(u_{r} * v_{r}\right), \\
& t=t_{0}\left(t_{1} * t_{1}\right)\left(t_{2} * t_{2}\right) \cdots\left(t_{m} * t_{m}\right)\left(u_{1} * v_{1}\right)\left(u_{2} * v_{2}\right) \cdots\left(u_{r} * v_{r}\right),
\end{aligned}
$$

where $F:=F_{s}=F_{t}=\left\{\left(u_{1}, v_{1}\right),\left(u_{2}, v_{2}\right), \ldots,\left(u_{r}, v_{r}\right)\right\}$ is a mutually reduced R-set by Lemma 5.2 , so that neither $s$ nor $t$ contains redundant $a * a$ subterms, and all $s_{i}, t_{j}$ are in normal form relative to $\rightarrow F$. It is also clear that $s_{0}=t_{0}$ because $s_{0}$ can be transformed into $t_{0}$ by a sequence of -replacements (and their reverse); hence $s_{0} \leftrightarrow_{F}^{*} t_{0}$ and then irreducibility gives $s=N_{F}(s)=N_{F}(t)=t$. It remains to show that the $a * a$ subterms are identical in both.

Consider a particular sequence of elementary transformations that converts $s$ into $t$ :

$$
s=s^{(0)} \mapsto s^{(1)} \mapsto s^{(2)} \mapsto \cdots \mapsto s^{(n)}=t .
$$

To make our analysis more transparent, we are going to give a pictorial interpretation of this sequence. Each subterm of $s^{(i)}$ with the form $(u * v)$ will be called a block. We let $B_{i}$ denote the set of blocks in $s^{(i)}$, including repeats. While we want to be able to distinguish between repeats in $B_{i}$ by subscripting the blocks, it is also notationally convenient for us to suppress these subscripts. We now let $G_{i}$ denote the set $\left\{b^{(i)}: b \in B_{i}\right\}$. So for example, under the form $s^{(0)}$ is presented above, we have

$$
G_{0}=\left\{\left(s_{1} * s_{1}\right)_{1}^{(0)}, \cdots,\left(s_{k} * s_{k}\right)_{k}^{(0)},\left(u_{1} * v_{1}\right)_{k+1}^{(0)}, \cdots,\left(u_{r} * v_{r}\right)_{k+r}^{(0)}\right\}
$$

(where here we chose to include the subscripts). We now let $G$ be the directed graph on $\cup_{0 \leq i \leq n} G_{i}$ with (directed) edges joining $a^{(i)}$ to $b^{(i+1)}$ if the block, $a$, of $s^{(i)}$ becomes the block $b$ of $s^{(i+1)}$. Because we have distinguished repeated blocks in each $s^{(i)}$, the graph is a disjoint union of directed paths. A complete path (that is, not part of some larger path) in this graph has its beginning in either $s^{(0)}$, or at a point at which a redundant block is introduced, and dually for the finish of a path.

Lemmas 5.4, 5.6 and the definition of redundancy removal give us the following useful fact.

Lemma 5.8. If $(e * f)^{(i)}$ and $(g * h)^{(j)}$ lie on the same path, then $e \leftrightarrow_{F}^{*} f \leftrightarrow_{F}^{*} g \leftrightarrow_{F}^{*} h$.

Let us fix some $\ell \leq k$. We show that $s_{\ell} * s_{\ell}$ appears in $t$. First we give an induction argument to show that for each $i \leq n$ there is (modulo commutativity of $*$ ) a block $g * h$ (for $g, h \in S^{*}$ ) of $s^{(i)}$ with the property that $h \leftrightarrow_{F}^{*} w s_{\ell}$ for some $w \in S^{*}$. This is certainly true in $s^{(0)}$ with $g=h=s_{\ell}$ and $w=1$. Now suppose the claim holds true for some $i<n$, with block $u * v$. So there is a word $w \in S^{*}$ such that $v \leftrightarrow_{F}^{*} w s_{\ell}$. If there is an edge in $G$ from $(u * v)^{(i)}$ to $\left(u^{\prime} * v^{\prime}\right)^{(i+1)}$, then Lemma 5.8 shows that the block $\left(u^{\prime} * v^{\prime}\right)$ of $s^{(i+1)}$ has the desired property $v^{\prime} \leftrightarrow_{F}^{*} v \leftrightarrow_{F}^{*} w s_{\ell}$. Otherwise, $(u * v)^{(i)}$ is the end of a path, and so $u * v$ is redundant in $s^{(i)}$ and removed in the transition to $s^{(i+1)}$. Hence also $u=v$. At this point there are apparently two possibilities: there is a block $w^{\prime} v * b$ in $s^{(i)}$ (distinct from $u * v$ ), where $w^{\prime}, b \in S^{*}$; or the semigroup part of $s^{(i+1)}$ has the form $w^{\prime} v$. This second case however cannot occur-indeed we would have $w^{\prime} v \leftrightarrow_{F}^{*} w^{\prime} w s_{\ell}$ and then Lemma 5.3 would show that $s_{0}=N_{F}\left(w^{\prime} v\right)=w^{\prime} w s_{\ell}$, contradicting irreducibility. So there is a block $w^{\prime} v * b$ in $s^{(i)}$. But then $w^{\prime} v \leftrightarrow_{F}^{*} w^{\prime} w s_{\ell}$ and the induction step holds.

The above argument shows in particular that $t$ contains a block $g * h$ in which $h \leftrightarrow_{F}^{*} w s_{\ell}$ for some $w \in S^{*}$. However, Lemma 5.3 shows that $N_{F}(g)=N_{F}(h)=w s_{\ell}$ and then irreducibility shows that one of $g$ or $h$ actually equals $w s_{\ell}$. We want to show that $w=1$ and $g=h$; that is, that $s_{\ell} * s_{\ell}$ is a block of $t$. By symmetry this will show that $s$ and $t$ are identical. 
Now $g * h$ cannot be one of the $u_{i} * v_{i}$ blocks, because these also appear in $s$, and then $s_{\ell} * s_{\ell}$ would be redundant in $s$, contradicting irreducibility. Hence $g * h$ is one of the $t_{i} * t_{i}$ terms and so $g=h=w s_{\ell}$. By symmetry we may repeat all of the above arguments starting with the block $w s_{\ell} * w s_{\ell}=t_{i} * t_{i}$ of $t$ and working towards $s$. Thus, we find that $w s_{\ell} * w s_{\ell}$ appears in $s$, or there is a block $w^{\prime} w s_{\ell} * w^{\prime} w s_{\ell}$ of $s$. However $s$ is irreducible and contains the block $s_{\ell} * s_{\ell}$. It follows that $w s_{\ell} * w s_{\ell}$ (or $w^{\prime} w s_{\ell} * w^{\prime} w s_{\ell}$, if that block occurs in $s$ ) and $s_{\ell} * s_{\ell}$ are in fact the same block. Therefore $w^{\prime} w=1$ and so $w=1$, as required. Thus symmetry gives $s=t$.

Thus a given $s \in T_{S}$ has a unique irreducible $t \in T_{S}$ to which it is congruent modulo $\approx$. Here is an algorithm to find it (see also Example 5.12 below). Given

$$
s=s_{0}\left(s_{1} * s_{1}\right)\left(s_{2} * s_{2}\right) \cdots\left(s_{k} * s_{k}\right)\left(u_{1} * v_{1}\right)\left(u_{2} * v_{2}\right) \cdots\left(u_{r} * v_{r}\right):
$$

- First step. Obtain the mutually reduced R-set $G_{s}$ from $F_{s}$, giving rise to the sequence of R-sets

$$
F_{s}=F_{0} \mapsto F_{1} \mapsto F_{2} \mapsto \cdots \mapsto F_{k}=G_{s},
$$

each related to the next by a transformation by replacement. For each such sequence of transformations, there is an obvious sequence of elementary transformations of $s$ and the terms it becomes, namely a sequence of $*$-replacements, giving rise to the sequence

$$
s=s^{(0)} \mapsto s^{(1)} \mapsto \cdots \mapsto s^{(k)}=t,
$$

so that for each $i, F_{i}=F_{s}(i)$. Thus $(a, b)$ being replaced by $(c, d)$ in passing from $F_{j}$ to $F_{j+1}$ corresponds to $(a * b)$ being replaced by $(c * d)$ in passing from $s^{(j)}$ to $s^{(j+1)}$, the only exception being when $c=d$ in which case the R-set loses an element, while $c * c$ goes "out the front" in $s^{(j+1)}$ with the other $\left(s_{i} * s_{i}\right)$ terms. At completion of this step, we have

$$
\begin{array}{r}
s^{\prime}=s_{0}\left(s_{1} * s_{1}\right)\left(s_{2} * s_{2}\right) \cdots\left(s_{k} * s_{k}\right)\left(c_{1} * c_{1}\right)\left(c_{2} * c_{2}\right) \cdots\left(c_{m} * c_{m}\right) \\
\left(x_{1} * y_{1}\right)\left(x_{2} * y_{2}\right) \cdots\left(x_{p} * y_{p}\right),
\end{array}
$$

where $G_{s}=\left\{\left(x_{1}, y_{1}\right),\left(x_{2}, y_{2}\right), \cdots,\left(x_{p}, y_{p}\right)\right\}, p \leq r$.

- Second step. Apply left to right $*$-replacement to the $\left(s_{i} * s_{i}\right)$ and $\left(c_{j} * c_{j}\right)$ (using the $\left.x_{i} * y_{i}\right)$ wherever possible, and --replacement to $s_{0}$, to give

$$
s^{\prime \prime}=s_{0}^{\prime}\left(b_{1} * b_{1}\right)\left(b_{2} * b_{2}\right) \cdots\left(b_{q} * b_{q}\right)\left(x_{1} * y_{1}\right)\left(x_{2} * y_{2}\right) \cdots\left(x_{p} * y_{p}\right) .
$$

- Third step. Eliminate redundancies where possible: if any $b_{i}$ appears as the right-hand portion of any other semigroup word appearing in $s^{\prime \prime}$, eliminate it. The result is $t=s_{0}^{\prime}\left(d_{1} *\right.$ $\left.d_{1}\right)\left(d_{2} * d_{2}\right) \cdots\left(d_{l} * d_{l}\right)\left(x_{1} * y_{1}\right)\left(x_{2} * y_{2}\right) \cdots\left(x_{p} * y_{p}\right)$, where now no redundancies exist.

This element $t$ is clearly irreducible and $t \approx s$, so it is the unique normal form of $s$, and we have our solution to the equational problem.

Corollary 5.9. The variety of twisted agreeable monoids has decidable equational theory.

We now want to obtain an algorithm for twisted agreeable semigroups. We claim that an identity $s \approx t$ in the language of agreeable semigroups holds in the variety of twisted agreeable semigroups if and only if it holds in the variety of twisted agreeable monoids. Certainly if $s \approx t$ holds on every twisted agreeable semigroup, it also holds on every twisted agreeable monoid. Now suppose that $s \approx t$ is not satisfied by some twisted agreeable semigroup $\mathbf{S}$. Recall from Proposition 2.3 that $\mathbf{S}$ embeds into the twisted agreeable semigroup $\mathcal{P}_{S}$ of all partial maps over the set $S$. Then $\mathcal{P}_{S} \not \models s \approx t$ also. But $\mathcal{P}_{S}$ is also a twisted agreeable monoid. Hence $s \approx t$ is not an identity of the variety of twisted agreeable monoids either.

Corollary 5.10. The variety of twisted agreeable semigroups has decidable equational theory.

In fact one may adapt the proof we gave for the monoid case to the semigroup case by replacing occurrences of terms of the form $x * 1$ by $x *(x * x)$. This is because for monoids, we have $x * 1 \approx x(x * x) * 1 \approx(x * 1) x * x \approx(x * 1(x * x)) \approx x *(x * x)$.

Corollary 5.11. The variety of Garvac'kiu semirings has decidable equational theory. 
Proof. This is because the Garvac'kil semirings are precisely the class of subalgebras of reducts of twisted SLORC's to the operations of $\cdot$ and $\wedge$.

In the language of rewrite systems, what we have produced is a convergent term rewriting system for twisted agreeable monoid terms. Our system consists of the following stages:

- Stage 1: Convert an arbitrary term $\phi$ in the language of agreeable monoids into an equivalent element $s$ of $T_{S}$ using the process outlined in Section 3.

- Stage 2: Apply the algorithm just given to $s$, giving irreducible $t \in T_{S}$.

Then $t$ is the normal form of $\phi$.

To demonstrate our algorithm applied to SLORC's and Garvac'kil semirings, we look at the Garvac'kiü identity 20 (of Section 2):

$$
x(a \wedge b \wedge c) \wedge y(b \wedge c) \approx x(a \wedge c) \wedge y(b \wedge c) .
$$

Recall from Example 3.1 that the left hand side of this identity is the agreeable term $x a(a * b)(a(a *$ $b) * c)[x a(a * b)(a(a * b) * c) * y b(b * c)]$.

Example 5.12. The normal form for the term $x a(a * b)(a(a * b) * c)[x a(a * b)(a(a * b) * c) * y b(b * c)]$ of Example 3.1 is ya $(b * a)(c * a)(y a * x a)$.

Proof. Examples 3.1 and 5.1 show that $x a(a * b)(a(a * b) * c)[x a(a * b)(a(a * b) * c) * y b(b * c)]$ is equivalent to $x a(a * b)(a * c)(x a * y b)(b * c)$. This completes stage 1 of our rewrite system. For stage 2 , following the normal form procedure described above:

Step 1. By Example 4.8, the mutually reduced R-set corresponding to $x a(a * b)(a * c)(x a * y b)(b * c)$ is $\{(b, a),(c, a),(y a, x a)\}$, and one application of rule R1 was required $((a, a)$ was removed). Hence the process in step 1 produces the term $x a(a * a)(b * a)(c * a)(y a * x a)$.

Step 2. There are no --replacements.

Step 3. There is one redundancy. We get irreducible normal form $x a(b * a)(c * a)(y a * x a)$, as required.

For comparison, the reader may work through Examples 3.1, 4.8, 5.1 and 5.12 using the term on the right hand side of the Garvac'kiu identity. As expected, the same normal form will be obtained.

\section{INTERPRETING THE USUAL WORD PROBLEM}

We have shown above that the variety of twisted agreeables interprets a weakened form of the uniform word problem for semigroups, where replacement is only allowed on final segments of words; see Lemma 4.9 and Corollary 4.10. Moreover, we showed that the equational theory of twisted agreeable semigroups is essentially equivalent to this weakened uniform word problem, which we proved to be decidable (Corollary 4.10). To interpret the full uniform word problem (which is undecidable) in a variety, we just need to adjoin a law sufficently strong to be able to make replacements in arbitrary positions. We are going to fix a particular approach that is both succinct and general. Let $\mathcal{L}$ be a fixed signature that includes two binary multiplications, · and *. A variety in the signature $\mathcal{L}$ satisfying associativity of $\cdot$ will be called a deductive variety if it satisfies the law $x a y(a * b) \approx x b y(a * b)$ where $x$ and $y$ are possibly empty (so really we have four different identities) ${ }^{1}$. Even if - and $*$ are the only operations present, a deductive variety need not be a variety of agreeable semigroups; however, we will denote the variety of all deductive agreeable semigroups by D.

Lemma 6.1. The variety of agreeable semigroups satisfying $x C(y) \approx C(y) x C(y)$ is a deductive variety. Amongst agreeables with identity, this law defines D.

Proof. Using $x C(y) \approx C(y) x C(y)$ we have $x a y(a * b) \approx x a(a * b) y(a * b) \approx x b(a * b) y(a * b) \approx x b y(a * b)$, while in the presence of an identity element, the deductive law gives $x C(y) \approx 1 x(C(y) * 1) \approx$ $C(y) x(C(y) * 1) \approx C(y) x C(y)$.

\footnotetext{
${ }^{1}$ We can establish all of the remaining results of this paper even if we insist that $x$ and $y$ not be empty; however there are some efficiencies to be gained by the current approach.
} 
Recall that a semigroup quasi-identity is an expression of the form $\left(\&_{1 \leq i \leq n} u_{i} \approx v_{i}\right) \rightarrow u \approx v$ for some semigroup words $u_{1}, \ldots, u_{n}, v_{1}, \ldots, v_{n}, u, v$ (in the $n=0$ case we mean simply the identity $u \approx v)$.

Let $\Phi:=\left(\&_{1 \leq i \leq n} u_{i} \approx v_{i}\right) \rightarrow u \approx v$ be a semigroup quasi-identity. We let $X_{\Phi, \mathrm{L}}$ denote set of the variables appearing in $u$ but not in any of words $u_{1}, \ldots, u_{n}, v_{1}, \ldots, v_{n}, v$ and $X_{\Phi, \mathrm{R}}$, the set of variables appearing in $v$ but not in $u_{1}, \ldots, u_{n}, v_{1}, \ldots, v_{n}, u$. Let $X_{\Phi}=X_{\Phi, \mathrm{L}} \cup X_{\Phi, \mathrm{R}}$.

Definition 6.2. Let $\Phi:=\left(\&_{1 \leq i \leq n} u_{i} \approx v_{i}\right) \rightarrow u \approx v$ be a semigroup quasi-identity. Then $\Phi^{\circledast}$ is the identity

$$
u \cdot \prod_{1 \leq i \leq n}\left(u_{i} * v_{i}\right) \cdot \prod_{z \in X_{\Phi}} C(z) \approx v \cdot \prod_{1 \leq i \leq n}\left(u_{i} * v_{i}\right) \cdot \prod_{z \in X_{\Phi}} C(z)
$$

(where $\prod$ denotes multiplication with respect to $\cdot$ ).

For example, if $\Phi$ is $a b \approx a c \rightarrow b \approx c$ (the left cancellation law), the set $X_{\Phi}$ is empty and $\Phi^{\circledast}$ is the identity $b(a b * a c) \approx c(a b * a c)$. If $\Phi$ is the identity $a^{n} b \approx b$ (satisfied, say, by abelian groups of exponent $n$ ), we find that $X_{\Phi}=\{a\}$ and we get the identity $a^{n} b C(a) \approx b C(a)$.

Lemma 6.3. Let $\mathrm{V}$ be a deductive variety. If a semigroup quasi-identity $\Phi=\left(\&_{1 \leq i \leq n} u_{i} \approx v_{i}\right) \rightarrow$ $u \approx v$ is satisfied by the variety of semigroups then $\mathrm{V}=\Phi^{\circledast}$.

Proof. Because $\left(\&_{1 \leq i \leq n} u_{i} \approx v_{i}\right) \rightarrow u \approx v$ holds in the variety of semigroups, it follows that $u$ can be transformed into $v$ by successive replacements of the form $u_{i} \rightarrow v_{i}$ or $v_{i} \rightarrow u_{i}$. The deductive laws enable this to be written as an equational deduction from $u\left(u_{1} * v_{1}\right) \ldots\left(u_{n} * v_{n}\right) C\left(x_{1}\right) \ldots C\left(x_{m}\right)$ to $v\left(u_{1} * v_{1}\right) \ldots\left(u_{n} * v_{n}\right) C\left(x_{1}\right) \ldots C\left(x_{m}\right)$. We omit the details, but give an example. Consider the semigroup quasi-identity $\Phi:=a \approx a b \quad \& \quad b c c \approx c \rightarrow a c c \approx a c$. This holds in the variety of all semigroups because if $a=a b$ and $b c c=c$ in a semigroup then we get $a c c=a b c c=a c$ (underlines indicate which subwords are being replaced). The corresponding identity $\Phi^{\circledast}$ is $a c c(a * a b)(b c c * c) \approx$ $a c(a * a b)(b c c * c)$. A deduction of this from the deductive laws is as follows (here, the square brackets indicate which part of the term is being manipulated by the deductive laws):

$$
\begin{aligned}
{[a c c(a * a b)](b c c * c) } & \approx[a b c c(a * a b)(b c c * c)] \\
& \approx a c(a * a b)(b c c * c) .
\end{aligned}
$$

Lemma 6.3 gives only one half of the interpretation of semigroup word problems. To give a full interpretation of the uniform word problem for semigroups in a deductive variety $\mathrm{V}$, we need to show that if $\Phi$ is a quasi-identity failing on the variety of all semigroups, then $\Phi^{\circledast}$ fails in V. That is, for each such $\Phi$, we need an algebra in $\mathrm{V}$ on which $\Phi^{\circledast}$ fails. The following result summarises this in a general fashion.

Lemma 6.4. Let $\mathrm{V}$ be a deductive variety and let $\left\{\Phi_{i}: i \in \mathbb{N}\right\}$ be a recursive family of semigroup quasi-identities with $\mathcal{T}:=\left\{i \in \mathbb{N}: \Phi_{i}\right.$ holds on all semigroups $\}$ and $\mathcal{F}:=\mathbb{N} \backslash \mathcal{T}$. If there are recursively inseparable sets $A \subseteq \mathcal{T}$ and $B \subseteq \mathcal{F}$ such that for each $i \in B$ there is an algebra $\mathbf{A}_{i} \in \mathrm{V}$ such that $\mathbf{A}_{i} \not=\Phi_{i}^{\circledast}$, then $\mathrm{V}$ has undecidable equational theory.

Proof. The set $\left\{i \in \mathbb{N}: \mathrm{V}=\Phi_{i}^{\circledast}\right\}$ contains $A$ (by Lemma 6.3 ) but is disjoint from $B$ (because of the algebras $\mathbf{A}_{i}$ ) and therefore is non-recursive.

While we have expressed this lemma in terms of general deductive varieties, we are going to concentrate on deductive varieties of agreeable semigroups. For the remainder of this section, all deductive varieties will be assumed to have precisely two operations, $\cdot$ and $*$.

To extend Lemma 6.4 to the greatest range of agreeable semigroup varieties, we want to be able to find quasi-identities $\left\{\Phi_{i}: i \in \mathbb{N}\right\}$ satisfying the lemma in such a way that the algebras $\mathbf{A}_{i}$ can be chosen to be as simple as possible.

Let $\mathbf{S}$ be a monoid with zero. We may endow $\mathbf{S}$ with an agreeable $*$ by setting $x * y=1$ if $x=y \neq 0$ and 0 otherwise. An agreeable semigroup isomorphic to one obtained in this way will be 
called atomic (in the natural order, every non-zero element is an atom). If $\mathbf{S}$ is a monoid then we let $\mathbf{S}^{\circledast}$ denote the atomic agreeable formed over $\mathbf{S}$ with a new zero element adjoined.

Lemma 6.5. Let $\mathbf{S}$ be a monoid and let $\Phi$ be a semigroup quasi-identity. If $\mathbf{S}^{\circledast} \models \Phi^{\circledast}$ as an atomic agreeable, then $\mathbf{S} \models \Phi$.

Proof. Suppose $\mathbf{S} \not \models \Phi$, where $\Phi$ is $\left(\&_{1 \leq i \leq n} u_{i} \approx v_{i}\right) \rightarrow u \approx v$. So $\Phi$ fails under some assignment $\theta$. Now consider $\theta$ as an assignment into the atomic agreeable $\mathbf{S}^{\circledast}$. We have $\theta\left(u_{i}\right)=\theta\left(v_{i}\right) \in S$ for all $i$, as well as $C(\theta(z))=1$ for all variables $z$, while $\theta(u) \neq \theta(v)$. Hence $\Phi^{\circledast}$ fails on the atomic agreeable $\mathbf{S}^{\circledast}$ under $\theta$ (extended in its codomain to include 0 ), because

$$
\begin{aligned}
\theta\left(u\left(u_{1} * v_{1}\right) \ldots\left(u_{n} * v_{n}\right) \prod_{z \in X_{\Phi}} C(z)\right) & =\theta(u) \cdot 1 \neq \theta(v) \cdot 1 \\
& =\theta\left(v\left(u_{1} * v_{1}\right) \ldots\left(u_{n} * v_{n}\right) \prod_{z \in X_{\Phi}} C(z)\right) .
\end{aligned}
$$

We let $\mathrm{G}_{\text {fin }}$ denote the variety generated by the class $\left\{\mathbf{G}^{\circledast} \mid \mathbf{G}\right.$ is a finite group $\}$. The members of $G_{\text {fin }}$ are height-1 semilattices with respect to $\wedge$ and the bottom element is a multiplicative zero element. These structures are often called flat extensions of groups and are of some independent interest; see [13] for example.

Theorem 6.6. Let $\mathrm{V}$ be any deductive variety containing $\mathrm{G}_{\text {fin. }}$. Then $\mathrm{V}$ has undecidable equational theory.

Proof. We are going to use the undecidability of the uniform word problem for finite groups, established by Slobodskoir [21]. First note that every finitely presented group $\mathbf{U}=\langle X: R\rangle$ is also finitely presented as a semigroup by taking the generating set $X \cup\left\{a^{-1}: a \in X\right\}$ and by adjoining the laws $b b^{-1}=b^{-1} b$ and $b c^{-1} c=c^{-1} c b=b$ for all generators $b, c \in X$. Because of this, we will assume that all groups are given semigroup presentations. The proof of the uniform word problem for finite groups can be described in the following way ([21]; see also [14]). There are recursively inseparable subsets $A$ and $B$ of $\mathbb{N}$, a finitely presented group $\mathbf{U}$ and a recursive list $\left\{u_{i}: i \in \mathbb{N}\right\}$ of words in the generators of $\mathbf{U}$ with the following two properties:

(1) If $n \in A$ then $u_{0}=u_{n}$ in $\mathbf{U}$;

(2) If $n \in B$ then $u_{0} \neq u_{n}$ and there is a finite group $\mathbf{G}(n)$ and homomorphism $\phi_{n}: \mathbf{U} \rightarrow \mathbf{G}(n)$ such that $\phi\left(u_{0}\right) \neq \phi\left(u_{n}\right)$.

We fix this group $\mathbf{U}$ (with presentation $\langle X: R\rangle$, say). For an instance $w=w^{\prime}$ of the word problem of $\mathbf{U}$, we let $\Phi_{w, w^{\prime}}$ be the quasi-identity $\left(\&_{u=v \in R} u \approx v\right) \rightarrow w \approx w^{\prime}$. Now let $\mathbf{V}$ be in the statement of the theorem. We use Lemma 6.4.

If $n \in A$ then $u_{0}=u_{n}$ in $\mathbf{U}$ by Property (1). Then $\Phi_{u_{0}, u_{n}}$ is satisfied by every semigroup. If $n \in B$ then the finite group $\mathbf{G}(n)$ fails $\Phi_{u_{0}, u_{n}}$ by Property (2). By Lemma 6.5, $\mathbf{G}(n)^{\circledast} \in \mathrm{V}$ fails $\Phi_{u_{0}, u_{n}}^{\circledast}$ as an atomic agreeable. Because $A$ and $B$ are recursively inseparable, Lemma 6.4 shows that $\checkmark$ has undecidable equational theory.

Let $\langle X: R\rangle$ be a semigroup presentation with undecidable word problem and with $X=$ $\left\{a_{1}, \ldots, a_{n}\right\}$ and $R$ finite. The injective homomorphism from $\left\{a_{1}, \ldots, a_{n}\right\}^{+}$to $\{a, b\}^{+}$defined by $a_{i} \mapsto a b^{i} a$ produces a presentation over $\{a, b\}$ with undecidable word problem. We can now replicate the proof of Theorem 6.6 using arbitrary atomic agreeables instead of those formed from finite groups. We get the following result.

Theorem 6.7. The 2-variable equational theory of any deductive variety containing the class of all atomic agreeables is undecidable.

In fact it follows from [8] that there is a finitely presented group on two generators with undecidable word problem. Under our translation to semigroup presentations, this group will have 
four generators. Thus the 4 -variable equational theory of any deductive variety containing all atomic agreeables over groups with 0 is undecidable.

As observed in [12], an atomic agreeable over a group with 0 is a twisted agreeable semigroup. Furthermore it satisfies the central law $x C(y) \approx C(y) x$. Let TC denote the variety of twisted central agreeables. The RC-semigroup reducts of members of TC generate the variety of twisted RCsemigroups with central closed elements. This variety was examined by Fountain [5] who showed that (after translation from right type-A semigroups to the language of RC-semigroups) it has decidable equational theory. Theorem 6.6 gives us the following contrasting result.

Corollary 6.8. Every variety of agreeable semigroups in the interval [TC, D] has undecidable equational theory.

We note here that there is a similarity between the methods in this section and those of [15]; in particular, both approaches use operations - and $*$ to encode the undecidability of the quasiequational theory of semigroups to obtain a large interval of varieties with undecidable equational theory. However, while the algebras of [15] are also semilattice ordered, the required identities involving $*$ are quite different to those used here.

\section{Semilattice ORDERED INVERSE SEMigroups}

We now apply Lemma 6.4 to the naturally semilattice ordered inverse semigroups, or inverse algebras of Leech. As discussed in Section 2, these are inverse semigroups with an additional semilattice operation whose order coincides with that of the natural inverse semigroup order; they become twisted SLORC's when given the term operation $C(x):=x^{-1} x$, and therefore they can also be interpreted as twisted agreeable but with the extra operation of inversion. There are many naturally occurring examples of these algebras; in particular the natural ordering on the inverse semigroup of all local automorphisms of an algebra is a semilattice ordering (see [16] for example). We will show that the variety of naturally semilattice ordered Clifford semigroups has undecidable equational theory.

For completeness, we begin with a self-contained examination of the axioms for this class. By a semilattice ordered semigroup we will mean a semigroup with additional $\wedge$ operation satisfying the usual semilattice laws on $\wedge$ and the implications $x \lesssim y \rightarrow x z \lesssim y z$ and $x \lesssim y \rightarrow z x \lesssim z y$ (where $a \lesssim b$ is an abbreviation for the expression $a \wedge b \approx a)$. Note that this class forms a variety since the two implications can be written as identities (for example, the first implication is equivalent to the identity $(a \wedge b) c \wedge b c \approx(a \wedge b) c)$. Every inverse semigroup has an associated partial order that is preserved by left and right multiplication; namely that given by $x \leq y$ if $x=y x^{-1} x$ (equivalently, $x=x x^{-1} y$; many other equivalent formulations can be found in [9] for example). We will say that a semilattice ordered inverse semigroup $\left\langle S ; \cdot, \wedge,{ }^{-1}\right\rangle$ is naturally semilattice ordered if its natural partial order agrees with the order induced by $\wedge$. This class forms a variety-for example, Proposition 3.1 of [12] shows that it can be defined by adjoining the laws $(x \wedge y) z^{-1} z \approx x z^{-1} z \wedge y z^{-1} z$ and $x(x \wedge y)^{-1}(x \wedge y) \approx x \wedge y$ to the above list. The following lemma shows that these two laws by themselves guarantee that the standard inverse agreeable semigroup is semilattice ordered in a stronger sense; namely that it satisfies the left and right distributivity laws and so forms a kind of semiring.

Lemma 7.1. Let $\mathbf{S}:=\left\langle S ; \cdot, \wedge,,^{-1}\right\rangle$ be an inverse semigroup with a semilattice operation $\wedge$. If $\mathbf{S}$ satisfies $(x \wedge y) z^{-1} z \approx x z^{-1} z \wedge y z^{-1} z$ and $x(x \wedge y)^{-1}(x \wedge y) \approx x \wedge y$ then $\mathbf{S}$ satisfies $(x \wedge y) z \approx x z \wedge y z$ and $z(x \wedge y) \approx z x \wedge z y$.

Proof. The standard closure $C(x):=x^{-1} x$ on $\mathbf{S}$ makes it a twisted RC-semigroup and the corresponding order is closed under left and right products and agrees with the natural order on $\mathbf{S}$ as an inverse semigroup [11]. As $\mathbf{S}$ satisfies $(x \wedge y) C(z) \approx x C(z) \wedge y C(z)$ and $x C(x \wedge y) \approx x \wedge y$, Proposition 3.1 of [12] shows that the order given by $\wedge$ agrees with that given by $C$ and so $\mathbf{S}$ is a SLORC. We first show that $\mathbf{S} \models(x \wedge y) z \approx x z \wedge y z$, which will show that $\mathbf{S}$ is term equivalent to a twisted agreeable semigroup [12]. 
Now $x, y, z \in S$ be such that $x \wedge y \leq x$ and $x \wedge y \leq y$. By right multiplication by $z$ we get $(x \wedge y) z \leq x z \wedge y z$. Next, let $w \in S$ be less than or equal to $x z$ and also $y z$. So $w=x z w^{-1} w$ and $w=y z w^{-1} w$ in $\mathbf{S}$ (equivalently, $w=x z C(w)$ and $w=y z C(w)$ ).

We have the following equalities in $\mathbf{S}$ :

$$
\begin{aligned}
(x \wedge y) z w^{-1} w & \left.=(x \wedge y) z\left(x z w^{-1} w\right)^{-1} x z w^{-1} w \quad \text { (by } w=x z C(w)\right) \\
& =(x \wedge y) z w^{-1} w z^{-1} x^{-1} x z w^{-1} w .
\end{aligned}
$$

Also, $\left(z w^{-1} w z^{-1}\right)^{2}=z w^{-1} w z^{-1} z w^{-1} w z^{-1}=z z^{-1} z w^{-1} w w^{-1} w z^{-1}=z w^{-1} w z^{-1}$, meaning that $z w^{-1} w z^{-1}$ is closed (because every idempotent is closed in $\mathbf{S}$ ). Therefore we may use distributivity of closed elements over $\wedge$ from the right (this is law $\left.(a \wedge b) c^{-1} c \approx a c^{-1} c \wedge b c^{-1} c\right)$ :

$$
\begin{aligned}
(x \wedge y) z w^{-1} w z^{-1} x^{-1} x z w^{-1} w & =\left(x z w^{-1} w z^{-1} \wedge y z w^{-1} w z^{-1}\right) x^{-1} x z w^{-1} w \\
& =\left(w z^{-1} \wedge w z^{-1}\right) x^{-1} x z w^{-1} w=w z^{-1} x^{-1} x z w^{-1} w \\
& =w w^{-1} w z^{-1} x^{-1} x z w^{-1} w=w\left(x z w^{-1} w\right)^{-1}\left(x z w^{-1} w\right) \\
& =w w^{-1} w=w .
\end{aligned}
$$

Hence $(x \wedge y) z \geq w$ and so $(x \wedge y) z \geq x z \wedge y z$ also.

For left distributivity, we may use the fact that $\mathbf{S}$ is also a left closure semigroup with left closure $C_{L}(x):=x x^{-1}$ and the corresponding order agrees with that of the right closure (both are the natural order) and hence with $\wedge$. Hence the laws $C_{L}(z)(x \wedge y) \approx C_{L}(z) x \wedge C_{L}(z) y$ and $C_{L}(x \wedge y) x \approx x \wedge y$ must automatically hold on $\mathbf{S}$ and we can use symmetry.

This lemma shows that if an inverse semigroup with the usual closure is agreeable, then it is twisted agreeable. Examples in [12] show that in general a twisted RC-semigroup whose closure makes it agreeable need not be twisted-agreeable (the law 17: $(a * b) c \approx c(a c * b c)$ may fail). Also, the algorithm given in Section 5 shows that the variety of all twisted agreeable semigroups does not satisfy the left distributivity rule $z(x \wedge y) \approx z x \wedge z y$ (in the $*$-notation, $z x(x * y) \approx z x(z x * z y)$ ).

It is well known that the inverse semigroups with central idempotents are exactly the class of Clifford semigroups, that is, semilattices of groups (see [9], for example). Axioms for naturally semilattice ordered Clifford semigroups are those guaranteed by Lemma 7.1 and the central idempotent law $y x^{-1} x \approx x^{-1} x y$. This class is studied in more detail in Leech, where a construction is given for the free naturally semilattice ordered Clifford semigroup starting from a free inverse Clifford semigroup. The following result is perhaps slightly surprising.

Theorem 7.2. The variety of naturally semilattice ordered Clifford semigroups has undecidable equational theory.

Proof. We view naturally semilattice ordered Clifford semigroups as agreeable Clifford semigroups and apply Lemma 6.4. First note that this class is indeed deductive because closed elements are central. As in the proof of Theorem 6.6, we now use the atomic agreeables over finite groups (with adjoined zero element), but this time allow the operation of inverse to be included. These are all agreeable Clifford semigroups. We can now apply Lemma 6.4 in an identical way to the proof of Theorem 6.6.

This paper has a sequel [10] in which the results of these later sections are put into a far more general perspective. In these last sections we have interpreted quasi-identities as identities. In fact there is a very obedient translation of the universal Horn logic of arbitrary partial algebras into the equational logic of varieties generated by certain semilattice ordered algebras (including naturally semilattice ordered Clifford semigroups and members of the variety TC).

\section{REFERENCES}

[1] A. Batbedat, $\gamma$-demi-groups, demi-modules, produit demi-directs, in Semigroups, Proceedings, Oberwolfalch, Germany 1978, Lecture Notes in Mathematics 855, Springer-Verlag (1981), 1-18.

[2] A. Batbedat and J. B. Fountain, Connections between left adequate semigroups and $\gamma$-semigroups, Semigroup Forum 22 (1981), 59-65. 
[3] B. Buchberger and R. Loos, Algebraic Simplification, in Computer Algebra. Symbolic and Algebraic Computation (ed. B. Buchberger, B.G. Collins, R. Loos), Springer-Verlag, New York, 11-43 (1983).

[4] W. Dudek and V. Trokhimenko, Functional Menger P-algebras, Comm. Algebra 30 (2002), 5921-5931.

[5] J. Fountain, Free right type A semigroups, Glasgow Math. J. 33 (1991), 135-148.

[6] V.S. Garvac'kiı̌, $\cap$-semigroups of transformations, Theory of Semigroups and its Applications, No. 2, pp.2-13, Izdat. Saratov. Uni., Saratov, 1971 [Russian].

[7] G. Gomes and V. Gould, Proper weakly left ample semigroups, Internat. J. Algebra Comput. 9 (1999), 721-739.

[8] G. Higman, B.H. Neumann and H. Neumann, Embedding theorems for groups, J. London Math. Soc. 24 (1949), $247-254$.

[9] J. Howie. "Fundamentals of Semigroup Theory" London Mathematical Society Monographs, Oxford University Press, 2nd ed., 1995.

[10] M. Jackson, Flattening a quasivariety, in progress.

[11] M. Jackson and T. Stokes, An invitation to C-semigroups, Semigroup Forum 62 (2001), 279-310.

[12] M. Jackson and T. Stokes, Agreeable semigroups, J. Algebra 266 (2003), 393-417.

[13] J. Ježek, M. Maróti and R. McKenzie, Quasiequational theories of flat algebras, Czech. Math. J. 55 (2005), 665-675.

[14] O. Kharlampovich and M. Sapir, Algorithmic problems in varieties, Internat. J. Algebra Comput. 5 (1995), 379-602.

[15] Á. Kurucz, I. Németi, I. Sain and A. Simon, Undecidable varieties of semilattice-ordered semigroups, of Boolean algebras with operators, and logics extending Lambek calculus, Bull. IGPL 1 (1993), 91-98.

[16] J. Leech, Inverse monoids with a natural semilattice ordering, Proc. London Math. Soc. (3) 70 (1995), 146-182.

[17] J. Leech, On the foundations of inverse monoids and inverse algebras, Proc. Edinburgh Math. Soc. (2) 41 (1998), $1-21$.

[18] B. Schein, Relation algebras and function semigroups, Semigroup Forum 1 (1970), 1-62.

[19] B. Schein, Restrictively multiplicative algebras of transformations, Izv. Vysš. Učebn, Zaved. Matematika 1970 (1970) No. 4 (95), 91-102 [Russian].

[20] B. Schweizer and A. Sklar, Function systems, Math. Annalen 172 (1967), 1-16.

[21] A. Slobodskor, Undecidability of the universal theory of finite groups Algebra i Logika 20 (1981) 207-230, 251 [Russian; English translation in Algebra and Logic 20 (1981), 139-156 (1982)].

[22] V. Trokhimenko, Menger's function systems, Izv. Vyss. Ucebn. Zaved. Matematika 1973, no. 11(138), 71-78 [Russian].

Department of Mathematics, La Trobe University, Victoria 3086, Australia

E-mail address: m.g.jackson@latrobe.edu.au

Mathematics, University of Waikato, Hamilton, New Zealand

E-mail address: stokes@math.waikato.ac.nz 